Bull. Soc. math. France

133 (1), 2005, p. 121-144

\title{
CHARACTERIZATION OF CYCLE DOMAINS VIA KOBAYASHI HYPERBOLICITY
}

\author{
By Gregor Fels \& Alan Huckleberry
}

\begin{abstract}
A real form $G$ of a complex semi-simple Lie group $G^{\mathbb{C}}$ has only finitely many orbits in any given $G^{\mathbb{C}}$-flag manifold $Z=G^{\mathbb{C}} / Q$. The complex geometry of these orbits is of interest, e.g., for the associated representation theory. The open orbits $D$ generally possess only the constant holomorphic functions, and the relevant associated geometric objects are certain positive-dimensional compact complex submanifolds of $D$ which, with very few well-understood exceptions, are parameterized by the Wolf cycle domains $\Omega_{W}(D)$ in $G^{\mathbb{C}} / K^{\mathbb{C}}$, where $K$ is a maximal compact subgroup of $G$. Thus, for the various domains $D$ in the various ambient spaces $Z$, it is possible to compare the cycle spaces $\Omega_{W}(D)$.

The main result here is that, with the few exceptions mentioned above, for a fixed real form $G$ all of the cycle spaces $\Omega_{W}(D)$ are the same. They are equal to a universal domain $\Omega_{A G}$ which is natural from the the point of view of group actions and which, in essence, can be explicitly computed.

The essential technical result is that if $\widehat{\Omega}$ is a $G$-invariant Stein domain which contains $\Omega_{A G}$ and which is Kobayashi hyperbolic, then $\widehat{\Omega}=\Omega_{A G}$. The equality of the cycle domains follows from the fact that every $\Omega_{W}(D)$ is itself Stein, is hyperbolic, and contains $\Omega_{A G}$.
\end{abstract}

Texte reçu le 12 février 2003, révisé le 14 octobre 2003

Gregor Fels, Fakultät für Mathematik, Universität Tübingen, Auf der Morgenstelle 14, 72076 (Germany) • E-mail : gfels@uni-tuebingen.de • Url : www.mathematik.unituebingen.de

Alan HuCkleberRY, Fakultät für Mathematik, Ruhr-Universität Bochum, Universitätsstrasse 150, 44801 Bochum (Germany) • E-mail : ahuck@cplx.ruhruni-bochum.de - Url : www.cplx.ruhr-uni-bochum.de

2000 Mathematics Subject Classification. - 22E46, 32M05.

Key words and phrases. - Complex geometry, cycles spaces, Lie groups, Schubert varieties. G.F.: Research supported by a Habilitationsstipendium from the Deutsche Forschungsgemeinschaft.

A.H.: Research partially supported by grants from the Deutsche Forschungsgemeinschaft and the Japanese Society for the Promotion of Science.

BULLETIN DE LA SOCIÉTÉ MATHÉMATIQUE DE FRANCE

(C) Société Mathématique de France

$0037-9484 / 2005 / 121 / \$ 5.00$ 
RÉSUMÉ (Caractérisation de domaines de cycles par l'hyperbolicité au sens de Kobayashi)

Une forme réelle $G$ d'un groupe de Lie semi-simple $G^{\mathbb{C}}$ n'admet qu'un nombre fini d'orbites dans toute $G^{\mathbb{C}}$-variété de drapeaux $Z=G^{\mathbb{C}} / Q$. La géométrie complexe de ces orbites est intéressante, par exemple pour la théorie de la représentation associée. Les fonctions holomorphes sur les orbites ouvertes $D$ de $G$ sont constantes en général; les objets géométriques importants liés à ces orbites sont des sous-variétés complexes de $D$ de dimension positives qui, à quelques rares exceptions bien comprises, sont paramétrées par les domaines de cycles de Wolf $\Omega_{W}(D) \in G^{\mathbb{C}} / K^{\mathbb{C}}$, où $K$ est un sous-groupe maximal compact de $G$. Alors, pour les domaines $D$ dans les variétés ambiantes $Z$, il est possible de comparer les domaines de cycles $\Omega_{W}(D)$.

Le résultat principal de cet article, aux exceptions près mentionnées ci-dessus, est que pour une forme réelle $G$ fixée, les domaines $\Omega_{W}(D)$ sont les mêmes. Ils sont égaux à un domaine universel $\Omega_{A G}$, qui est canonique du point de vue d'actions de groupe et qui peut être essentiellement calculé.

Le résultat technique important est que tout domaine de Stein hyperbolique au sens de Kobayashi $\widehat{\Omega}$ qui contient $\Omega_{A G}$ est égal à $\Omega_{A G}$. L'égalité des domaines de cycles s'ensuit du fait que chaque $\Omega_{W}(D)$ est lui-même de Stein, hyperbolique et contient $\Omega_{A G}$.

\section{Introduction}

Let $G$ be a non-compact real semi-simple Lie group which is embedded in its complexification $G^{\mathbb{C}}$ and consider the associated $G$-action on a $G^{\mathbb{C}}$-flag manifold $Z=G^{\mathbb{C}} / Q$. It is known that $G$ has only finitely many orbits in $Z$; in particular, there exit open $G$-orbits $D$. In each such open orbit every maximal compact subgroup $K$ of $G$ has exactly one orbit $C_{0}$ which is a complex submanifold (see [42]).

Let $q:=\operatorname{dim}_{\mathbb{C}} C_{0}$, regard $C_{0}$ as a point in the space $\mathcal{C}^{q}(Z)$ of $q$-dimensional compact cycles in $Z$ and let

$$
\Omega:=G^{\mathbb{C}} \cdot C_{0}
$$

be the orbit in $\mathcal{C}^{q}(Z)$. Define the Wolf cycle space $\Omega_{W}(D)$ to be the connected component of $\Omega \cap \mathcal{C}^{q}(D)$ which contains the base cycle $C_{0}$.

Since the above mentioned basic paper [42], there has been a great deal of work aimed at describing these cycle spaces. Even in situations where good matrix models are available this is not a simple matter. Using a variety of techniques, exact descriptions of $\Omega_{W}(D)$ have been given in a number of special situations (see e.g. [1] [4], [3], [13], [17], [23], [24], [34], [38], [41], [45]).

In [16] it was conjectured that, except in the holomorphic Hermitian case where $\Omega_{W}(D)$ is just the associated bounded symmetric space, the cycle spaces can be naturally identified with a certain universal domain $\Omega_{A G}$ which only depends on $G$. This domain, which is precisely defined below, is a certain $G$ invariant neighborhood of the Riemannian symmetric space $M=G / K$ in its complexification $\Omega=G^{\mathbb{C}} / K^{\mathbb{C}}$. 
The inclusion $\Omega_{A G} \subset \Omega_{W}(D)$ was proved in most cases in [17] by analyzing concrete models and by using a nice general result which reduces this inclusion to special cases.

In [25], using incidence geometry given by Schubert varieties (see also [23] and [22]), it was shown that $\Omega_{W}(D)$ agrees with the Schubert domain $\Omega_{S}(D)$ which is defined by removing certain algebraic incidence divisors from $\Omega$.

The Schubert domains in turn contain a universal domain $\Omega_{I}$ which is known to agree with $\Omega_{A G}$. The inclusion $\Omega_{A G} \subset \Omega_{I}$ was proved by complex analytic methods (see [22]), but now there is an algebraic proof (see [33]) which may be more appropriate, because the situation would apriori seem to be algebraic in nature. The inclusion $\Omega_{I} \subset \Omega_{A G}$ was shown in [2]. Thus,

$$
\Omega_{A G} \subset \Omega_{I} \subset \Omega_{S}(D)=\Omega_{W}(D) .
$$

In particular $\Omega_{A G} \subset \Omega_{W}(D)$, has now been proved in complete generality. Therefore, to prove the above mentioned conjecture it is necessary to prove the opposite inclusion $\Omega_{W}(D) \subset \Omega_{A G}$.

This is a consequence of the following complex geometric characterization of $\Omega_{A G}$ which is the main result of the present paper (see Theorem 3.4.5).

THEOREM 1.0.1. - If $\widehat{\Omega}$ is a G-invariant domain which contains $\Omega_{A G}$ in $\Omega$ and which is in addition Stein and Kobayashi hyperbolic, then $\widehat{\Omega}=\Omega$.

Obviously $\widehat{\Omega}=\Omega_{W}(D)$ is $G$-invariant. It follows directly from the definitions that Schubert domains are Stein (see [25]). Thus $\Omega_{W}(D)=\Omega_{S}(D)$ implies that the cycle spaces are Stein, a fact that has been known in the measurable case for some time (see [43]).

Using a slight refinement of the results in [22], we show here that, with the exception of the holomorphic Hermitian case where $\Omega_{W}(D)$ is just the associated bounded symmetric space, $\Omega_{W}(D)$ is naturally embedded in $\Omega$ as a Kobayashi hyperbolic domain.

Consequently, with this well-understood exception, the above theorem together with the inclusion $\Omega_{A G} \subset \Omega_{W}(D)$ shows that $\Omega_{W}(D)=\Omega_{A G}$.

Before going to the main body of our work, let us set the notation.

Let $M=G / K$ be the associated Riemannian symmetric space of nonpositive curvature embedded in $M^{\mathbb{C}}=G^{\mathbb{C}} / K^{\mathbb{C}}$ as an orbit of the same base point $x_{0}$ as was chosen above in the discussion of cycle spaces.

Denote by $\theta$ a Cartan involution on $\mathfrak{g}^{\mathbb{C}}$ which restricts to a Cartan involution on $\mathfrak{g}$ such that $\operatorname{Fix}\left(\theta_{\mid \mathfrak{g}}\right)=\mathfrak{k}$ is the Lie algebra of the given maximal compact subgroup $K$. The anti-holomorphic involution $\sigma: \mathfrak{g}^{\mathbb{C}} \rightarrow \mathfrak{g}^{\mathbb{C}}$ which defines $\mathfrak{g}$ commutes with $\theta$ as well as with the holomorphic extension $\tau$ of $\theta_{\mid \mathfrak{g}}$ to $\mathfrak{g}^{\mathbb{C}}$.

Let $\mathfrak{u}$ be the fixed point set of $\theta$ in $\mathfrak{g}^{\mathbb{C}}, U$ be the associated maximal compact subgroup of $G^{\mathbb{C}}$ and define $\Sigma$ to be the connected component containing $x_{0}$ of $\left\{x \in U \cdot x_{0}: G_{x}\right.$ is compact $\}$. Set $\Omega_{A G}:=G \cdot \Sigma$. 
To cut down on the size of $\Sigma$, one considers a maximal Abelian subalgebra $\mathfrak{a}$ in $\mathfrak{p}$ (where $\mathfrak{g}=\mathfrak{k} \oplus \mathfrak{p}$ is the Cartan decomposition of $\mathfrak{g}$ ) and notes that $G \cdot(\exp (i \mathfrak{a}) \cap \Sigma) \cdot x_{0}=\Omega_{A G}$.

In fact there is an explicitly defined neighborhood $\omega_{A G}$ of $0 \in \mathfrak{a}$ such that $i \omega_{A G}$ is mapped diffeomorphically onto its images $\exp \left(i \omega_{A G}\right)$ and $\exp \left(i \omega_{A G}\right) \cdot x_{0}$ and $\Omega_{A G}=G \cdot \exp \left(i \omega_{A G}\right) \cdot x_{0}$.

The set $\omega_{A G}$ is defined by the set of roots $\Phi(\mathfrak{a})$ of the adjoint representation of $\mathfrak{a}$ on $\mathfrak{g}$ : It is the connected component containing $0 \in \mathfrak{a}$ of the set which is obtained from $\mathfrak{a}$ by removing the root hyperplanes $\left\{\mu=\frac{1}{2} \pi\right\}$ for all $\mu \in \Phi$. It is convex and is invariant under the action of the Weyl group $\mathcal{W}(\mathfrak{a})$ of the symmetric space $G / K$.

Modulo $\mathcal{W}(\mathfrak{a})$, the set $\exp \left(i \omega_{A G}\right) \cdot x_{0}$ is a geometric slice for the $G$-action on $\Omega_{A G}$. From this root point of view, $\Sigma$ can be seen to be the set of points which are at most half way from $x_{0}$ to the cut-point locus in the compact Riemannian symmetric space $U / K$ (see [11]).

\section{Spectral properties of $\Omega_{\mathrm{AG}}$}

2.1. Linearization. - The map

$$
\eta: G^{\mathbb{C}} \longrightarrow \operatorname{Aut}_{\mathbb{R}}\left(\mathfrak{g}^{\mathbb{C}}\right), \quad x \longmapsto \sigma \circ \operatorname{Ad}(x) \circ \tau \circ \operatorname{Ad}\left(x^{-1}\right),
$$

provides a suitable linearization of the setting at hand. The idea of using this linearization in the context of double coset spaces is due to T. Masuki. Some of the results in this and the following section on the Jordan decomposition can be found in $\S 4$ of [32]. In particular, in $\S 3.2$ for the sake of completeness we give proofs of his Proposition 3 and Proposition 4. In this section elementary properties of $\eta$ are summarized.

Let $G^{\mathbb{C}}$ act on $\operatorname{Aut}_{\mathbb{R}}\left(\mathfrak{g}^{\mathbb{C}}\right)$ by $h \cdot \varphi:=\operatorname{Ad}(h) \circ \varphi \circ \operatorname{Ad}\left(h^{-1}\right)$.

Lemma 2.1.1 (G-equivariance). - For $h \in G$ it follows that $\eta(h \cdot x)=h \cdot \eta(x)$ for all $x \in G^{\mathbb{C}}$.

Proof. - By definition $\eta(h \cdot x)=\sigma \operatorname{Ad}(h) \operatorname{Ad}(x) \tau \operatorname{Ad}\left(x^{-1}\right) \operatorname{Ad}\left(h^{-1}\right)$. Since $h$ belongs to $G$, it follows that $\sigma$ and $\operatorname{Ad}(h)$ commute and the desired result is immediate.

The normalizer of $K^{\mathbb{C}}$ in $G^{\mathbb{C}}$ is denoted by $N^{\mathbb{C}}:=N_{G^{\mathbb{C}}}\left(K^{\mathbb{C}}\right)$. It is indeed the complexification of $N:=N_{U}(K)$.

Lemma 2.1.2 ( $N^{\mathbb{C}}$-invariance). - The map $\eta$ factors through a $G$-equivariant embedding of $G^{\mathbb{C}} / N^{\mathbb{C}}$ :

$$
\eta(x)=\eta(y) \Longleftrightarrow y=x g^{-1} \text { for some } g \in N^{\mathbb{C}} .
$$


Proof. - We may write $y=x g^{-1}$ for some $g \in G^{\mathbb{C}}$. Thus it must be shown that $\eta(x)=\eta\left(x g^{-1}\right)$ if and only if $g \in N^{\mathbb{C}}$. But $\eta(x)=\eta\left(x g^{-1}\right)$ is equivalent to $\operatorname{Ad}(g) \tau=\tau \operatorname{Ad}(g)$, which, in turn, is equivalent to the fact that $\operatorname{Ad}(g)$ stabilizes the complexified Cartan decomposition $\mathfrak{g}^{\mathbb{C}}=\left(\mathfrak{g}^{\mathbb{C}}\right)^{\tau} \oplus\left(\mathfrak{g}^{\mathbb{C}}\right)^{-\tau}=\mathfrak{k}^{\mathbb{C}} \oplus \mathfrak{p}^{\mathbb{C}}$.

Now, if $\operatorname{Ad}(g)$ stabilizes $\mathfrak{k}^{\mathbb{C}} \oplus \mathfrak{p}^{\mathbb{C}}$, then $\operatorname{Ad}(g)\left(\mathfrak{k}^{\mathbb{C}}\right)=\mathfrak{k}^{\mathbb{C}}$, i.e., $g \in N^{\mathbb{C}}$. On the other hand, given any $g \in N^{\mathbb{C}}$, it follows $\operatorname{Ad}(g)\left(\mathfrak{p}^{\mathbb{C}}\right)=\mathfrak{p}^{\mathbb{C}}$, because $\mathfrak{p}^{\mathbb{C}}$ is the orthogonal complement of $\mathfrak{k}^{\mathbb{C}}$ with respect to the Killing form of $\mathfrak{g}^{\mathbb{C}}$.

Note that $N^{\mathbb{C}} / K^{\mathbb{C}}$ is a finite Abelian group (see [15] for a classification). Consequently, up to finite covers, $\eta$ is an embedding of the basic space $G^{\mathbb{C}} / K^{\mathbb{C}}$.

The involutions $\sigma$ and $\tau$ are regarded as acting on $\operatorname{Aut}_{\mathbb{R}}\left(\mathfrak{g}^{\mathbb{C}}\right)$ by conjugation. On $\operatorname{Im}(\eta)$ their behavior is particularly simple.

Lemma 2.1.3 (Action of the basic involutions). - For all $x \in G^{\mathbb{C}}$ it follows that

1) $\eta(\tau(x))=\tau(\eta(x))$,

2) $\sigma(\eta(x))=\eta(x)^{-1}$,

In particular $\operatorname{Im}(\eta)$ is both $\sigma$ - and $\tau$-invariant.

Proof. - Let $\varphi_{*}: \mathfrak{g}^{\mathbb{C}} \rightarrow \mathfrak{g}^{\mathbb{C}}$ denote the differential of $\varphi: G^{\mathbb{C}} \rightarrow G^{\mathbb{C}}$ and $\operatorname{Int}(x): G^{\mathbb{C}} \rightarrow G^{\mathbb{C}}$ be defined by $\operatorname{Int}(x)(z):=x z x^{-1}$. The first statement follows directly from the facts that $\sigma$ and $\tau$ commute and

$$
\tau \operatorname{Ad}(x) \tau=(\tau \operatorname{Int}(x) \tau)_{*}=\operatorname{Int}(\tau(x))_{*}=\operatorname{Ad}(\tau(x)) .
$$

For the second statement note that $\sigma \eta(x)=\operatorname{Ad}(x) \tau \operatorname{Ad}\left(x^{-1}\right)$, and thus $\eta(x) \sigma \eta(x)=\sigma$.

We have seen that $\eta$ is a $G$-equivariant map which induces a finite equivariant map $\eta: G^{\mathbb{C}} / K^{\mathbb{C}} \rightarrow \operatorname{Aut}_{\mathbb{R}}\left(\mathfrak{g}^{\mathbb{C}}\right)$. We will shortly see that the image $\eta\left(G^{\mathbb{C}} / K^{\mathbb{C}}\right)$ is also closed in $\operatorname{Aut}_{\mathbb{R}}\left(\mathfrak{g}^{\mathbb{C}}\right)$. Hence, for a characterization of $G$-orbits in $G^{\mathbb{C}} / K^{\mathbb{C}}$ and their topological properties we may identify $G^{\mathbb{C}} / K^{\mathbb{C}}$ with its image in $\operatorname{Aut}_{\mathbb{R}}\left(\mathfrak{g}^{\mathbb{C}}\right)$ on which $G$ acts by conjugation.

The following special case of a general result on conjugacy classes (see [26, p. 117] and [7]) is of basic use.

Lemma 2.1.4. - Let $V$ be a finite-dimensional $\mathbb{R}$-vector space, $H$ a closed reductive algebraic subgroup of $\mathrm{GL}_{\mathbb{R}}(V)$ and $s \in \mathrm{GL}_{\mathbb{R}}(V)$ an element which normalizes $H$. Regard $H$ as acting on $\mathrm{GL}_{\mathbb{R}}(V)$ by conjugation. Then, for a semi-simple $s$ the orbit $H \cdot s$ is closed.

Corollary 2.1.5. - The image $\operatorname{Im}(\eta)$ is closed in $\operatorname{Aut}_{\mathbb{R}}\left(\mathfrak{g}^{\mathbb{C}}\right)$.

Proof. - It is enough to show that $G^{\mathbb{C}} \cdot \tau=\left\{\operatorname{Ad}(g) \tau \operatorname{Ad}\left(g^{-1}\right): g \in G^{\mathbb{C}}\right\}$ is closed in $\operatorname{Aut}_{\mathbb{C}}\left(\mathfrak{g}^{\mathbb{C}}\right)$. Since $\tau$ is semi-simple and normalizes $G^{\mathbb{C}}$ in this representation, this follows from Lemma 2.1.4. 
Using a bit of invariant theory over $\mathbb{R}$, we are able to carry over the standard result on orbits in the complex case (see [32, Prop. 4]).

Proposition 2.1.6. - If $\eta(x)=s$ is semi-simple, then $G \cdot x$ is closed.

Proof. - It is enough to show that $G \cdot s$ is closed. By Lemma 2.1.4 the complex orbit $G^{\mathbb{C}} \cdot s$ is closed. Define $\hat{\sigma}: \operatorname{Aut}_{\mathbb{R}}\left(\mathfrak{g}^{\mathbb{C}}\right) \rightarrow \operatorname{Aut}_{\mathbb{R}}\left(\mathfrak{g}^{\mathbb{C}}\right)$ by $\hat{\sigma}(\varphi)=(\sigma(\varphi))^{-1}$. Here $\sigma$ acts by conjugation as usual.

Now $\operatorname{Im}(\eta)$ belongs to the fixed point set $\operatorname{Fix}(\hat{\sigma})$ and since $G^{\mathbb{C}} \cdot s \cap \operatorname{Fix}(\hat{\sigma})$ consists of only finitely many $G$-orbits (see [9]), it follows that $G \cdot s$ is closed.

2.2. Jordan Decomposition. - Here $x$ denotes an arbitrary element of $G^{\mathbb{C}}$ and $s u=u s=\eta(x)$ is its Jordan decomposition in $\mathrm{GL}_{\mathbb{R}}\left(\mathfrak{g}^{\mathbb{C}}\right)$. Since $\operatorname{Aut}_{\mathbb{R}}\left(\mathfrak{g}^{\mathbb{C}}\right)$ is algebraic, $s, u \in \operatorname{Aut}_{\mathbb{R}}\left(\mathfrak{g}^{\mathbb{C}}\right)$ as well. If $\eta(x)=u s$ is not semi-simple, i.e., $u \neq 1$, consider $\xi=\log (u) \in \operatorname{End}_{\mathbb{R}}\left(\mathfrak{g}^{\mathbb{C}}\right)$. Since $\xi$ is nilpotent, $t \mapsto \exp (t \xi)$ is an algebraic map and $\exp (\mathbb{Z} \xi) \subset \operatorname{Aut}_{\mathbb{R}}\left(\mathfrak{g}^{\mathbb{C}}\right)$. It follows that $\exp (t \xi) \in \operatorname{Aut}_{\mathbb{R}}\left(\mathfrak{g}^{\mathbb{C}}\right)$ for all $t \in \mathbb{R}$. In particular, $u$ is in the connected component $\operatorname{Aut}_{\mathbb{R}}\left(\mathfrak{g}^{\mathbb{C}}\right)^{0}$, and $\xi$ is a derivation: $\xi=\operatorname{ad}(N)$ for some nilpotent $N \in \mathfrak{g}^{\mathbb{C}}$. Finally, $u=\operatorname{Ad}(\exp (N))=\exp (\operatorname{ad} N)$.

Given an element $z \in \operatorname{Aut}_{\mathbb{R}}\left(\mathfrak{g}^{\mathbb{C}}\right)$, let $\left(\mathfrak{g}^{\mathbb{C}}\right)^{z}=\left\{X \in \mathfrak{g}^{\mathbb{C}}: z(X)=X\right\}$ denote the subalgebra of fixed points. Observe also that if $\nu: \mathfrak{g}^{\mathbb{C}} \rightarrow \mathfrak{g}^{\mathbb{C}}$ is any involution such that $\nu(z)=z$ or $\nu(z)=z^{-1}$, then the subalgebra $\left(\mathfrak{g}^{\mathbb{C}}\right)^{z}$ is $\nu$-stable. For $z$ semi-simple the subalgebra $\left(\mathfrak{g}^{\mathbb{C}}\right)^{z}$ is reductive.

Proposition 2.2.1 (Lifting of the Jordan decomposition)

For $x \in G^{\mathbb{C}}$ with Jordan decomposition $\eta(x)=u \cdot s$ there exists a nilpotent element $N \in\left(\mathfrak{g}^{\mathbb{C}}\right)^{s} \cap i \mathfrak{g}$ such that

1) $u=\operatorname{Ad}(\exp (N))$

2) $\eta\left(\exp \left(\frac{1}{2} N\right) \cdot x\right)=s$.

Proof. - Let $N \in \mathfrak{g}^{\mathbb{C}}$ be the element with $u=\operatorname{Ad}(\exp (N))$ as explained above. First we show that $N \in\left(\mathfrak{g}^{\mathbb{C}}\right)^{s} \cap i \mathfrak{g}$. From Lemma 2.1.3(2) it follows that $\sigma(\eta(x))=\sigma(u s)=s^{-1} u^{-1}$. This implies $\sigma(u)=u^{-1}$ or, equivalently, $\sigma(N)=-N$, i.e., $N \in i \mathfrak{g}$. Secondly, the statement that $\operatorname{Ad}(\exp (N))$ commutes with $s$ is equivalent to $s \mathrm{e}^{\operatorname{ad}(N)} s^{-1}=\mathrm{e}^{\operatorname{ad}(N)}$ which is the same as $s(N)=N$ in the semi-simple case. Thus $N \in\left(\mathfrak{g}^{\mathbb{C}}\right)^{s} \cap i \mathfrak{g}$.

Finally, since $N \in\left(\mathfrak{g}^{\mathbb{C}}\right)^{s}$, it follows that $\operatorname{Ad}(\exp (t N))$ commutes with $s$ for all $t \in \mathbb{R}$. Having also in mind that $\sigma(N)=-N$, it follows that

$$
\begin{aligned}
\eta\left(\exp \frac{1}{2} N \cdot x\right) & =\sigma \operatorname{Ad}\left(\exp \frac{1}{2} N\right) \operatorname{Ad}(x) \tau \operatorname{Ad}\left(x^{-1}\right) \operatorname{Ad}\left(\exp -\frac{1}{2} N\right) \\
& =\operatorname{Ad}\left(\exp -\frac{1}{2} N\right) \cdot \sigma \operatorname{Ad}(x) \tau \operatorname{Ad}\left(x^{-1}\right) \cdot \operatorname{Ad}\left(\exp -\frac{1}{2} N\right) \\
& =\operatorname{Ad}\left(\exp -\frac{1}{2} N\right) \cdot s u \cdot \operatorname{Ad}\left(\exp -\frac{1}{2} N\right) \\
& =s \cdot \operatorname{Ad}\left(\exp -\frac{1}{2} N\right) \cdot u \cdot \operatorname{Ad}\left(\exp -\frac{1}{2} N\right)=s
\end{aligned}
$$

TOME $133-2005-\mathrm{N}^{\mathrm{O}} 1$ 
Observe now that since $\sigma(s)=s^{-1},\left(\mathfrak{g}^{\mathbb{C}}\right)^{s}$ is a $\sigma$-stable reductive subalgebra. Let $\left(\mathfrak{g}^{\mathbb{C}}\right)^{s}=\mathfrak{h} \oplus \mathfrak{q}$ be its $\sigma$-eigenspace decomposition. We now build an appropriate $\mathfrak{s l}_{2}$-triple $(E, H, F)$ around $N=E$ in $\left(\mathfrak{g}^{\mathbb{C}}\right)^{s}$.

Lemma 2.2.2. - Let $E \in\left(\mathfrak{g}^{\mathbb{C}}\right)^{s} \cap i \mathfrak{g}$ be an arbitrary non-trivial nilpotent element. There exists an $\mathfrak{s l}_{2}$-triple $(E, H, F)$ in $\left(\mathfrak{g}^{\mathbb{C}}\right)^{s}$, i.e.,

$$
[E, F]=H, \quad[H, E]=2 E \quad \text { and } \quad[H, F]=-2 F
$$

such that $E, F \in \mathfrak{q}$ and $H \in \mathfrak{h}$.

Proof. - Since $\left(\mathfrak{g}^{\mathbb{C}}\right)^{s}$ is reductive, there exists a $\mathfrak{s l}_{2}$-triple $(E, H, F)$ in $\left(\mathfrak{g}^{\mathbb{C}}\right)^{s}$ by the theorem of Jacobson-Morozov. It can be chosen to be $\sigma$-compatible.

To see this, split $H=H^{\sigma}+H^{-\sigma}$ with respect to the $\sigma$-eigenspace decomposition of $\left(\mathfrak{g}^{\mathbb{C}}\right)^{s}$. Since $[H, E]=2 E$ and $\sigma(E)=-E$, it follows that

$$
\left[H^{-\sigma}, E\right]=0 \text {. }
$$

Hence, we may assume that $H=H^{\sigma}$ (see [8, Chap. VIII, §11, Lemme 6]). Observe further that in this case one has $[E, F]=\left[E,(F)^{-\sigma}\right]=H$ and $\left[H,(F)^{-\sigma}\right]=(F)^{-\sigma}$. The desired result follows then from the uniqueness of the third element $F$ in a $\mathfrak{s l}_{2}$-triple.

Now we have all the ingredients which are needed to give a complete characterization of the closed orbits in $\operatorname{Im}(\eta)$ (see [32, Prop. 3]):

Proposition 2.2.3 (Closed orbits). - If $\eta(x)=u s$ is the Jordan decomposition, then the orbit $G \cdot \eta(x)=G \cdot(s u)$ contains the closed orbit $G \cdot s$ in its closure $\overline{G \cdot \eta(x)}$. In particular, $G \cdot \eta(x)$ is closed if and only if $\eta(x)$ is semisimple and $s \in \operatorname{Im}(\eta)$.

Proof. - Let $u=\operatorname{Ad}(\exp N)$ with $N$ as in Proposition 2.2.1. Hence, by Lemma 2.2.2 there is a $\mathfrak{s l}_{2}$-triple $(N, H, F)(E=N)$ such that $[t H, N]=2 t N$, i.e., $\operatorname{Ad}(\exp t H)(N)=\mathrm{e}^{2 t} N$ for every $t \in \mathbb{R}$. Note also that $\exp (\mathbb{R} H) \subset$ $G \cap \exp \left(\mathfrak{g}^{\mathbb{C}}\right)^{s}$ by construction of the $\mathfrak{s l}_{2}$-triple. It follows that

$$
\begin{aligned}
\eta(\exp t H \cdot x) & =\exp t H \cdot(u s)=\operatorname{Ad}(\exp t H) \cdot u s \cdot \operatorname{Ad}(\exp -t H) \\
& =\operatorname{Ad}(\exp t H) \cdot u \cdot \operatorname{Ad}(\exp -t H) \cdot s \\
& =\operatorname{Ad}(\exp t H) \operatorname{Ad}(\exp N) \operatorname{Ad}(\exp -t H) \cdot s \\
& =\operatorname{Ad}\left(\exp \mathrm{e}^{2 t} N\right) \cdot s
\end{aligned}
$$

For $t \rightarrow-\infty$ it follows $\lim \exp t H \cdot(u s)=\operatorname{Ad}\left(\exp \mathrm{e}^{2 t} N\right) \cdot s=s$. Hence, the closed orbit $G \cdot s$ lies in the closure of $G \cdot(u s)$. In particular $G \cdot(u s)$ is non-closed if $u \neq 1$, i.e., if $\eta(x)$ is not semi-simple. This, together with Proposition 2.1.6 implies that $G \cdot \eta(x)$ is closed if and only if $\eta(x)$ is semi-simple. Recall that the image $\operatorname{Im}(\eta)$ is closed. This forces $s \in \operatorname{Im}(\eta)$ and the proof is now complete. 
2.3. Elliptic elements and closed orbits. - Every non-zero complex number $z$ has the unique decomposition $r \cdot \mathrm{e}^{i \phi}$ into the hyperbolic part $r>0$ and elliptic part $\mathrm{e}^{i \phi}$. This generalizes for an arbitrary semi-simple element $s \in \mathrm{GL}\left(\mathfrak{g}^{\mathbb{C}}\right)$ : By decomposing its eigenvalues one obtains the unique decomposition $s=s_{\text {ell }} \cdot s_{\text {hyp }}=s_{\text {hyp }} \cdot s_{\text {ell }}$. An element $x \in G^{\mathbb{C}}$ is said to be elliptic if $\eta(x)=s$ is semi-simple with eigenvalues lying in the unit circle. It should be remarked that $x$ itself may in such a case not be a semi-simple element of the group $G^{\mathbb{C}}$, e.g., $K^{\mathbb{C}}$ contains unipotent elements.

Let $\Omega_{\text {ell }} \subset G^{\mathbb{C}}$ be the set of elliptic elements. This set is invariant by the right-action of $K^{\mathbb{C}}$, and therefore by choosing the same base point $x_{0}$ as in the case of $\Omega_{\mathrm{AG}}$, by abuse of notation we also regard $\Omega_{\mathrm{ell}}$ as a subset of $G^{\mathbb{C}} / K^{\mathbb{C}}$. We reiterate that, since the map $\eta$ is not a group morphism, the classical notion of an elliptic element in $G^{\mathbb{C}}$ differs from the above definition.

Lemma 2.3.1. - For $U$ the maximal compact subgroup of $G^{\mathbb{C}}$ defined by $\theta$ it follows that $U \subset \Omega_{\mathrm{ell}}$.

Proof. - For $\theta$ the Cartan involution defining $\mathfrak{u}$, observe that

$$
\widehat{U}:=\left\{\varphi \in \operatorname{Aut}_{\mathbb{R}}\left(\mathfrak{g}^{\mathbb{C}}\right): \varphi \theta=\theta \varphi\right\}
$$

is a maximal compact subgroup of $\operatorname{Aut}_{\mathbb{R}}\left(\mathfrak{g}^{\mathbb{C}}\right)$ (with identity component $\operatorname{Ad}(U)$ ).

Now $\theta$ commutes with every term in the definition of $\eta(u)$ for every $u \in U$. It follows that $\theta \eta(u)=\eta(u) \theta$. Therefore $\eta(U)$ is contained in the compact group $\widehat{U}$ and consequently $U \subset \Omega_{\mathrm{ell}}$.

Proposition 2.3.2 (Elliptic elements). - In the homogeneous space $G^{\mathbb{C}} / K^{\mathbb{C}}$ the set of elliptic elements is described as $\Omega_{\mathrm{ell}}=G \cdot \exp (i \mathfrak{a}) \cdot x_{0}$.

Proof. - Observe that $\Omega_{\mathrm{ell}}$ is $G$-invariant. Hence, the above lemma implies that $G \cdot \exp (i \mathfrak{a}) \cdot x_{0} \subset \Omega_{\text {ell }}$.

Conversely, suppose $x$ is elliptic, i.e., $\eta(x)$ is contained in some maximal compact subgroup of $\operatorname{Aut}_{\mathbb{R}}\left(\mathfrak{g}^{\mathbb{C}}\right)$. Hence, there is a Cartan involution $\theta^{\prime \prime}: \mathfrak{g}^{\mathbb{C}} \rightarrow \mathfrak{g}^{\mathbb{C}}$ which commutes with $\eta(x)$. We now make the usual adjustments so that, after replacing $x$ by an appropriate $G$-translate, $\eta(x)$ will commute with the given Cartan involution $\theta$.

For this, if $\theta^{\prime \prime}$ does not commute with $\sigma$, define the semi-simple element $\rho:=$ $\sigma \theta^{\prime \prime} \sigma \theta^{\prime \prime}$ which is diagonalizable with all positive eigenvalues over $\mathbb{R}$. It follows that $\rho^{t}$ is defined for all $t \in \mathbb{R}$, and $\theta^{\prime}:=\rho^{\frac{1}{4}} \theta^{\prime \prime} \rho^{-\frac{1}{4}}$ commutes with $\sigma$ (see [21, Chap. III, §7]). By direct calculation one verifies that $\rho$, hence $\rho^{t}$ commutes with $\eta(x)$. Thus it follows that $\theta^{\prime}$ and $\eta(x)$ commute.

Finally, since $\theta^{\prime}$ and our original $\theta$ both commute with $\sigma$, there exists $h \in G$ such that $\operatorname{Ad}(h) \theta^{\prime} \operatorname{Ad}\left(h^{-1}\right)=\theta$. Consequently, if $x$ is replaced by $h^{-1} \cdot x$, then we may assume that $\eta(x)$ and $\theta$ commute. 
Now we will adjust $x$ so that it lies in $U$. With respect to the global Cartan decomposition of $G^{\mathbb{C}}$ defined by $\theta$ write $x=u \exp (Z)$, i.e., $u \in U$ and $\theta(Z)=-Z$. We now show that in fact $\exp (Z) \in K^{\mathbb{C}}$.

Since $\theta$ commutes with $\sigma, \tau$ and $u$ and anti-commutes with $Z$, we have

$$
\begin{aligned}
\theta \eta(x) & =\theta \cdot\left(\sigma \operatorname{Ad}(u) \operatorname{Ad}(\exp (Z)) \tau \operatorname{Ad}\left(\exp (-Z) \operatorname{Ad}\left(u^{-1}\right)\right)\right. \\
& =\sigma \operatorname{Ad}(u) \operatorname{Ad}(\exp (-Z)) \tau \operatorname{Ad}(\exp (Z)) \operatorname{Ad}\left(u^{-1}\right) \cdot \theta
\end{aligned}
$$

On the other hand

$$
\theta \eta(x)=\eta(x) \theta=\sigma \operatorname{Ad}(u) \operatorname{Ad}(\exp (Z)) \tau \operatorname{Ad}(\exp (-Z)) \operatorname{Ad}\left(u^{-1}\right) \cdot \theta .
$$

Combining these two equations, we obtain

$$
\operatorname{Ad}(\exp (Z)) \tau \operatorname{Ad}(\exp (-Z))=\operatorname{Ad}(\exp (-Z)) \tau \operatorname{Ad}(\exp (Z))
$$

and consequently $\operatorname{Ad}(\exp (2 Z))$ commutes with $\tau$. Since the restriction $\operatorname{Ad}: \exp (i \mathfrak{u}) \rightarrow \operatorname{Aut}\left(\mathfrak{g}^{\mathbb{C}}\right)$ is injective, it follows that $\tau(\exp (Z))=\exp (Z)$, i.e., $\exp (Z) \in K^{\mathbb{C}}$. Replacing $x$ by $x \exp (-Z)$, it follows that $x \cdot x_{0}=x \exp (-Z) \cdot x_{0}$; hence, we may assume that $x \in U$.

Since $U=K \cdot \exp (i \mathfrak{a}) \cdot K$, we may assume that $x \in K \exp (i \mathfrak{a})$ and then translate it by left multiplication by an element of $K$ to reach the following conclusion: If $x \in G^{\mathbb{C}}$ is elliptic, then there exists $h \in G$ and $\ell \in K^{\mathbb{C}}$ with $h x \ell$ in $\exp i \mathfrak{a}$ or, equivalently, there is $h \in G$ with $h x \cdot x_{0} \in \exp (i \mathfrak{a}) \cdot x_{0}$. This proves the inclusion $\Omega_{\text {ell }} \subset G \cdot \exp (i \mathfrak{a}) \cdot x_{0}$.

The following is a key ingredient for understanding the $G$-orbit structure in $\operatorname{bd}\left(\Omega_{A G}\right)$.

Proposition 2.3.3. - One has $\exp (i \mathfrak{a}) \cdot x_{0} \cap c \ell\left(\Omega_{A G}\right)=c \ell\left(\exp \left(i \omega_{A G}\right) \cdot x_{0}\right)$

Proof. - If $x \in \mathrm{c} \ell\left(\exp \left(i \omega_{A G}\right) \cdot x_{0}\right)$, then it is elliptic and therefore its orbit $G \cdot x$ is closed. In other words $\exp (i \mathfrak{a}) \cdot x_{0} \cap \mathrm{c} \ell\left(\Omega_{A G}\right) \supset \mathrm{c} \ell\left(\exp \left(i \omega_{A G}\right) \cdot x_{0}\right)$.

For the opposite inclusion, observe that if $s, s^{\prime} \in \exp (i \mathfrak{a}) \cdot x_{0}$ and $s^{\prime} \in G \cdot s$, then $s^{\prime}=k(s)$ for some element $k$ of the Weyl group. Thus, if $s$ belongs to $\mathrm{c} \ell\left(\exp \left(i \omega_{A G}\right) \cdot x_{0}\right)$, then $s^{\prime}$ belongs to $\mathrm{c} \ell\left(\exp \left(i \omega_{A G}\right) \cdot x_{0}\right)$ as well. Therefore, in order to prove the opposite inclusion it is enough to show that, given $s^{\prime} \in \exp \left(i \mathfrak{a} \cdot x_{0}\right) \cap \mathrm{c} \ell\left(\Omega_{A G}\right)$, there exists $s \in \mathrm{c} \ell\left(\exp \left(i \omega_{A G}\right) \cdot x_{0}\right)$ with $s^{\prime} \in G \cdot s$.

Given $s^{\prime}$ as above, there exist sequences $\left\{s_{n}\right\} \subset \exp \left(i \omega_{A G}\right) \cdot x_{0}$ and $\left\{s_{n}^{\prime}\right\} \subset$ $\Omega_{A G}$ such that $s_{n}^{\prime} \in G \cdot s_{n}, s_{n}^{\prime} \rightarrow s^{\prime}$ and $s_{n} \rightarrow s \in \mathrm{c} \ell\left(\exp \left(i \omega_{A G}\right) \cdot x_{0}\right)$. Consider the (real) categorical quotient map $\pi: \operatorname{Aut}_{\mathbb{R}}\left(\mathfrak{g}^{\mathbb{C}}\right) \rightarrow \operatorname{Aut}_{\mathbb{R}}\left(\mathfrak{g}^{\mathbb{C}}\right) / / G$. It is continuous, the base is Hausdorff and in every fiber there is exactly one closed $G$-orbit. Since $\pi\left(s_{n}\right)=\pi\left(s_{n}^{\prime}\right)$, it follows that $G \cdot s=G \cdot s^{\prime}$.

Corollary 2.3.4. - Let $\Omega_{c \ell}$ denote $\{x \in \Omega: G \cdot x$ is closed $\}$. Then

$$
\Omega_{c \ell} \cap c \ell\left(\Omega_{A G}\right)=G \cdot c \ell\left(\exp \left(i \omega_{A G}\right) \cdot x_{0}\right)=\Omega_{\mathrm{ell}} \cap c \ell\left(\Omega_{A G}\right) .
$$


Proof. - From Proposition 2.3.2, $\Omega_{A G} \subset \Omega_{\mathrm{ell}}$. By continuity, the semi-simple part of $\eta(x)$ is elliptic for every $x \in \mathrm{c} \ell\left(\Omega_{A G}\right)$. Thus $\Omega_{c \ell} \cap \mathrm{c} \ell\left(\Omega_{A G}\right) \subset \Omega_{\mathrm{ell}} \cap$ $\mathrm{c} \ell\left(\Omega_{A G}\right)$, because elements of closed orbits are semi-simple. Proposition 2.3.2 gives $\Omega_{c \ell} \cap \mathrm{c} \ell\left(\Omega_{A G}\right) \subset G \cdot \mathrm{c} \ell\left(\exp \left(i \omega_{A G}\right) \cdot x_{0}\right)$, and from Proposition 2.3.3 it follows that $G \cdot c \ell\left(\exp \left(i \omega_{A G}\right) \cdot x_{0}\right) \subset \Omega_{c \ell} \cap c \ell\left(\Omega_{A G}\right)$. So we have $\Omega_{c \ell} \cap c \ell\left(\Omega_{A G}\right) \subset$ $G \cdot \mathrm{c} \ell\left(\exp \left(i \omega_{A G}\right) \cdot x_{0}\right)=\Omega_{\mathrm{ell}} \cap \mathrm{c} \ell\left(\Omega_{A G}\right)$. Finally, if $x \in \Omega_{\mathrm{ell}}$, then in particular it is semi-simple and $G \cdot x$ is closed. This proves the remaining inclusion.

\section{3. $Q_{2}$-slices}

At a generic point $y \in \mathrm{bd}\left(\Omega_{\mathrm{AG}}\right)$ we determine a 3-dimensional, $\sigma$-invariant, semi-simple subgroup $S^{\mathbb{C}}$ such that $S=\left(S^{\mathbb{C}}\right)^{\sigma}=\operatorname{Fix}\left(\sigma: S^{\mathbb{C}} \rightarrow S^{\mathbb{C}}\right)$ is a noncompact real form and such that the isotropy group $S_{y}^{\mathbb{C}}$ is either a maximal complex torus or its normalizer. Geometrically speaking, $Q_{2}=S^{\mathbb{C}} \cdot y$ is either the 2-dimensional affine quadric, which can be realized by the diagonal action as the complement of the diagonal in $\mathbb{P}_{1}(\mathbb{C}) \times \mathbb{P}_{1}(\mathbb{C})$, or its (2-1)-quotient, which is defined by exchanging the factors and which can be realized as the complement of the (closed) 1-dimensional orbit of $\mathrm{SO}_{3}(\mathbb{C})$ in $\mathbb{P}_{2}(\mathbb{C})$. By abuse of notation, we refer in both cases to $S^{\mathbb{C}} \cdot y$ as a 2-dimensional affine quadric.

The key property is that, up to the above mentioned possibility of a (2-1)cover, the intersection $Q_{2} \cap \Omega_{\mathrm{AG}}$ is the Akhiezer-Gindikin domain in $S^{\mathbb{C}} / K_{S}^{\mathbb{C}}$ for the unit disk $S / K_{S}$.

For the sake of brevity we say that the orbit $S^{\mathbb{C}} \cdot y$ is a $Q_{2}$-slice at $y$ whenever it has all of the above properties.

3.1. Existence. - Given a non-closed $G$-orbit $G \cdot y$ in $\operatorname{bd}\left(\Omega_{\mathrm{AG}}\right)$, we may apply Proposition 2.2.1 to obtain a lifting of the semi-simple (elliptic) part of the Jordan decomposition of $\eta(x)$. For an appropriate base point $z$ this lifting can be chosen in $\operatorname{bd}\left(\exp \left(i \omega_{\mathrm{AG}}\right)\right)$. Recall that the action $G^{\mathbb{C}} \times \operatorname{Aut}_{\mathbb{R}}\left(\mathfrak{g}^{\mathbb{C}}\right) \rightarrow \operatorname{Aut}_{\mathbb{R}}\left(\mathfrak{g}^{\mathbb{C}}\right)$ is given by conjugation (see $\left.\S 3.1\right)$. Note that the isotropy Lie algebra at $\varphi \in \operatorname{Aut}_{\mathbb{R}}\left(\mathfrak{g}^{\mathbb{C}}\right)$ is the totally real subalgebra of fixed points $\left(\mathfrak{g}^{\mathbb{C}}\right)^{\varphi}=\left\{Z \in \mathfrak{g}^{\mathbb{C}}: \varphi(Z)=Z\right\}$.

Lemma 3.1.1 (Optimal base point). - Every non-closed G-orbit $G \cdot y$ in $\operatorname{bd}\left(\Omega_{\mathrm{AG}}\right)$ contains a point $z=\exp E \cdot \exp i A \cdot x_{0}$ such that $E \in\left(\mathfrak{g}^{\mathbb{C}}\right)^{\eta(\exp i A)} \cap i \mathfrak{g}$ is a non-trivial nilpotent element.

Proof. - Let $\eta(y)=s u$ be the Jordan decomposition and let $N \in\left(\mathfrak{g}^{\mathbb{C}}\right)^{s} \cap i \mathfrak{g}$ be as in Proposition 2.2.1. We then have

$\eta(\exp y)=\eta\left(\exp \left(-\frac{1}{2} N\right) \exp \left(\frac{1}{2} N\right) \cdot y\right)=\operatorname{Ad}(\exp N) \circ \eta\left(\exp \left(\frac{1}{2} N\right) \cdot y\right)=u \cdot s$. 
By Proposition 2.2.3 and Corollary 2.3.4 the semi-simple element $\eta\left(\exp \left(\frac{1}{2} N\right) \cdot y\right)$ is elliptic. Hence, Proposition 2.3.2 implies the existence of $g \in G$ and $A \in \operatorname{bd}\left(\omega_{\mathrm{AG}}\right)$ such that $\exp \frac{1}{2} N \cdot y=g^{-1} \exp i A \cdot x_{0}$.

Define now $E:=\operatorname{Ad}(g)\left(-\frac{1}{2} N\right)$ and observe that $g \cdot y=\exp E \exp i A \cdot x_{0}$. Finally, $E \in\left(\mathfrak{g}^{\mathbb{C}}\right)^{g \cdot s}=\left(\mathfrak{g}^{\mathbb{C}}\right)^{\eta(\exp i A)}$, and the lemma is proved.

Recall that $\left(\mathfrak{g}^{\mathbb{C}}\right)^{\eta(\exp i A)}$ is a $\sigma$-stable real reductive algebra. Let

$$
\left(\mathfrak{g}^{\mathbb{C}}\right)^{\eta(\exp i A)}=\mathfrak{h} \oplus \mathfrak{q}
$$

be the decomposition into $\sigma$-eigenspaces. In this notation, the nilpotent element $E$ as in the above lemma belongs to $\mathfrak{q}$.

Let now an arbitrary non-closed orbit $G \exp E \exp i A \cdot x_{0}$ be given. Fix a $\mathfrak{s l}_{2}$-triple $(E, H, F)$ as in Lemma 2.2.2. Let $S^{\mathbb{C}}$ be the complex subgroup of $G^{\mathbb{C}}$ defined by this triple. Set $e:=i E, f:=-i F$ and let $S$ be the $\sigma$-invariant real form in $S^{\mathbb{C}}$. The Lie algebra of $S$ is then the subalgebra generated by the $\mathfrak{s l}_{2}$ triple $(e, H, f)$. Finally, let $x_{1}=\exp (i A) \cdot x_{0}$ be the base point chosen as above in the closure of a given $G$-orbit.

Lemma 3.1.2. - The connected component $\left(S_{x_{1}}^{\mathbb{C}}\right)^{0}$ of the $S^{\mathbb{C}}$-isotropy at $x_{1}$ is the 1-parameter subgroup $\{\exp (z H): z \in \mathbb{C}\} \cong \mathbb{C}^{*}$.

Proof. - Since the action $S^{\mathbb{C}} \times G^{\mathbb{C}} / K^{\mathbb{C}} \rightarrow G^{\mathbb{C}} / K^{\mathbb{C}}$ is affine-algebraic, the orbit $S^{\mathbb{C}} \cdot \exp i A \cdot x_{0}=S^{\mathbb{C}} \cdot x_{1}$ is an affine variety. Then the isotropy at $\exp i A \cdot x_{0}$ is 1-dimensional or $S^{\mathbb{C}}$. Note that $S^{\mathbb{C}} \cdot x_{1}$ cannot be a point, because by construction $\exp E \cdot x_{1} \neq x_{1}$; therefore $S_{x_{1}}^{\mathbb{C}}$ is 1-dimensional.

We now show that $\exp \mathbb{R} H \cdot x_{1}=x_{1}$, or equivalently, $\exp t H \cdot \eta\left(x_{1}\right)=\eta\left(x_{1}\right)$. Define $\varphi:=\operatorname{Ad}(\exp (i A)) \tau \operatorname{Ad}(\exp (-i A))$ and note that $H \in\left(\mathfrak{g}^{\mathbb{C}}\right)^{\sigma} \cap\left(\mathfrak{g}^{\mathbb{C}}\right)^{\varphi}=\mathfrak{h}$ yields

$$
\begin{aligned}
\exp t H \cdot \eta\left(x_{1}\right) & =\operatorname{Ad}(\exp t H) \circ \sigma \varphi \circ \operatorname{Ad}(\exp -t H) \\
& =\operatorname{Ad}(\exp t H) \operatorname{Ad}(\exp -t H) \circ \sigma \varphi=\eta\left(x_{1}\right) .
\end{aligned}
$$

It follows that $\exp \mathbb{C} H \cdot x_{1}=x_{1}$. Since $S_{x_{1}}^{\mathbb{C}}$ is 1-dimensional and $H$ semi-simple, we deduce $\left(S_{x_{1}}^{\mathbb{C}}\right)^{0}=\exp \mathbb{C} H \cong \mathbb{C}^{*}$.

3.2. Genericity. - Without going into a technical analysis of $b d\left(\Omega_{\mathrm{AG}}\right)$, we will construct $Q_{2}$-slices only at its generic points. The purpose of this section is to introduce the appropriate notion of "generic" and prove that the set of such points is open and dense. The set of generic points is defined to be the complement of the union of small semi-algebraic sets $\mathcal{C}$ and $\mathcal{E}$ in $\operatorname{bd}\left(\Omega_{\mathrm{AG}}\right)$. We begin with the definition of $\mathcal{C}$.

Let $R:=\operatorname{bd}\left(\exp \left(i \omega_{\mathrm{AG}}\right) \cdot x_{0}\right)$ and recall that for $y \in \operatorname{bd}\left(\Omega_{\mathrm{AG}}\right)$ the orbit $G \cdot y$ is closed if and only if $G \cdot y \cap R \neq \varnothing$. In fact $R$ parameterizes the closed orbits in $\operatorname{bd}\left(\Omega_{\mathrm{AG}}\right)$ up to the orbits of a finite group. Recall also that $R$ is naturally identified with bd $\left(\omega_{\mathrm{AG}}\right)$, which is the boundary of a convex polytope, and is 
defined by linear inequalities. Let $E$ be the image in $R$ of the lower-dimensional edges in $\operatorname{bd}\left(\omega_{\mathrm{AG}}\right)$, i.e., the set of points which are contained in at least two root hyperplanes $\left\{\alpha=c_{\alpha}\right\}$. Finally, let $R_{\text {gen }}:=R \backslash E$.

As we have seen in Corollary 2.3.4, the set of closed orbits in the boundary of $\Omega_{\mathrm{AG}}$ can be described as $G \cdot \operatorname{bd}\left(\exp \left(i \omega_{\mathrm{AG}}\right) x_{0}\right)$. This is by definition the set $\mathcal{C}$.

Lemma 3.2.1. - For $x \in R$ it follows that $\operatorname{dim} G \cdot x \leq \operatorname{codim}_{\Omega} \operatorname{bd}(\Omega)-2$.

Proof. - Note that $\mathrm{bd}\left(\Omega_{\mathrm{AG}}\right)$ is connected and of codimension 1 in $\Omega$. The $G$-isotropy group $C_{K}(\mathfrak{a})$ at generic points of $\exp \left(i \omega_{A G}\right) \cdot x_{0}$ fixes this slice pointwise and therefore is contained in a maximal compact subgroup of the isotropy subgroup $G_{x}$ of each of its boundary points. Since by definition $G_{x}$ is non-compact, it follows that $\operatorname{dim} G_{x}$ is larger than the dimension of the generic $G$-isotropy subgroup at points of $\exp \left(i \omega_{A G}\right) \cdot x_{0}$.

REMARK. - For $x \in \operatorname{bd}\left(\Omega_{\mathrm{AG}}\right)_{\text {gen }}$ the isotropy subgroup $G_{x}$ is precisely calculated in $\S 3.3$. This shows that $\operatorname{dim} G \cdot x=\operatorname{codim}_{\Omega} \operatorname{bd}\left(\Omega_{\mathrm{AG}}\right)-m$, where $m$ is at least 2 . Thus, by semi-continuity we have for all $x \in \mathrm{bd}\left(\Omega_{\mathrm{AG}}\right)$ the estimate

$$
\operatorname{dim} G \cdot x \leq \operatorname{codim}_{\Omega} \operatorname{bd}\left(\Omega_{\mathrm{AG}}\right)-m .
$$

Now let $X:=\operatorname{Im}(\eta) \subset \operatorname{Aut}_{\mathbb{R}}\left(\mathfrak{g}^{\mathbb{C}}\right)$. It is a connected component of a real algebraic submanifold in $\operatorname{Aut}_{\mathbb{R}}\left(\mathfrak{g}^{\mathbb{C}}\right)$. The complexification $X^{\mathbb{C}}$ of $X$ which is contained in the complexification $\operatorname{Aut}_{\mathbb{C}}\left(\mathfrak{g}^{\mathbb{C}} \times \mathfrak{g}^{\mathbb{C}}\right)$ of $\operatorname{Aut}_{R}\left(\mathfrak{g}^{\mathbb{C}}\right)$ is biholomorphic to $G^{\mathbb{C}} / N^{\mathbb{C}} \times G^{\mathbb{C}} / N^{\mathbb{C}}$, where $N^{\mathbb{C}}$ denotes the normalizer of $K^{\mathbb{C}}$ in $G^{\mathbb{C}}$. The complexification of the piecewise real analytic variety $R$ is a piecewise complex analytic subvariety $R^{\mathbb{C}}$ of $X^{\mathbb{C}}$ defined in a neighborhood of $R$ in $X^{\mathbb{C}}$. Finally, let $\pi: X^{\mathbb{C}} \rightarrow X^{\mathbb{C}} / / G^{\mathbb{C}}$ be the complex categorical quotient.

Recall that in every $\pi$-fiber there is a unique closed $G^{\mathbb{C}}$-orbit. The closed $G$ orbits in $X$ are components of the the real points of the closed $G^{\mathbb{C}}$-orbits which are defined over $\mathbb{R}$. For a more extensive discussion of the interplay between the real and complex points in complex varieties defined over $\mathbb{R}$ see [39], [40], [9].

Let $k:=\operatorname{dim}_{\mathbb{R}} \Omega-\operatorname{dim} R-m$ be the dimension of the generic $G$-orbits of points of $R$ and let $S_{k}$ be the closure in $X^{\mathbb{C}}$ of

$$
\left\{z \in X^{\mathbb{C}}: G^{\mathbb{C}} \cdot z \text { is closed and } k \text {-dimensional }\right\} .
$$

Define $\mathcal{C}_{k}:=S_{k} \cap R^{\mathbb{C}}$. It follows that $\mathcal{C}_{k}$ is a piecewise complex analytic set of dimension $k+\operatorname{dim}_{\mathbb{C}} R^{\mathbb{C}}$.

Proposition 3.2.2. - The set $G \cdot R=\left\{x \in \operatorname{bd}\left(\Omega_{\mathrm{AG}}\right): G \cdot x\right.$ is closed $\}$ is contained in a closed semi-algebraic subset $\mathcal{C}$ of codimension at least 1 in $\operatorname{bd}\left(\Omega_{\mathrm{AG}}\right)$.

Proof. - The set $\mathcal{C}$ is defined to be the intersection of the real points of $\mathcal{C}_{k}$ with $\operatorname{bd}\left(\Omega_{\mathrm{AG}}\right)$. The desired result follows from $\operatorname{dim}_{\mathbb{C}} \mathcal{C}_{k}=k+\operatorname{dim}_{\mathbb{C}} R^{\mathbb{C}}$. 
Recall that $\pi$ denotes the categorical quotient map $\pi: X^{\mathbb{C}} \rightarrow X^{\mathbb{C}} / / G^{\mathbb{C}}$. Define $\mathcal{E}:=\eta^{-1}\left(\pi^{-1}(\pi(E))\right) \cap \operatorname{bd}\left(\Omega_{\mathrm{AG}}\right)$. In particular it is a closed semialgebraic subset of $\mathrm{bd}\left(\Omega_{\mathrm{AG}}\right)$ which contains the set

$$
\left\{x \in \operatorname{bd}\left(\Omega_{\mathrm{AG}}\right): \mathrm{c} \ell(G \cdot x) \cap E \neq \varnothing\right\} .
$$

Definition. - A point $z \in \operatorname{bd}\left(\Omega_{\mathrm{AG}}\right)$ is said to be generic if it is contained in the complement of $\mathcal{C} \cup \mathcal{E}$.

Let $\operatorname{bdgen}_{\mathrm{gen}}\left(\Omega_{\mathrm{AG}}\right)$ denote the set of generic boundary points.

Proposition 3.2.3. - The set of generic points $\operatorname{bd}_{g e n}\left(\Omega_{\mathrm{AG}}\right)$ is open and dense in $\mathrm{bd}\left(\Omega_{\mathrm{AG}}\right)$.

It has already been noted that $\mathcal{C}$ and $\mathcal{E}$ are closed. Since $\mathcal{C}$ is of codimension two, the complement of $\mathcal{C}$ is dense. Thus this proposition is an immediate consequence of the following fact.

Proposition 3.2.4. - The saturation $\mathcal{E}$ is at least 1-codimensional in $\operatorname{bd}\left(\Omega_{\mathrm{AG}}\right)$.

This in turn follows from a computation of the dimension of the fibers at points of $E$ of the above mentioned categorical quotient. For this it is convenient to use the Jordan decomposition $\eta(z)=u \cdot s$ for $z \in \Omega$ such that $x=\exp (i \mathfrak{a}) \cdot x_{0}$ is in $\mathrm{c} \ell(G \cdot z)$.

As in Lemma 3.1.1 we choose an optimal base point such that $\eta(x)=s$ and $u=\operatorname{Ad}(\exp (N))$ with $N \in \mathfrak{q}$, where $\mathfrak{h} \oplus \mathfrak{q}$ is the $\sigma$-decomposition of $\mathfrak{l}=\left(\mathfrak{g}^{\mathbb{C}}\right)^{s}$. Let $N_{x}$ be the the cone of nilpotent elements in $\mathfrak{q}$ and observe that the saturation $\mathcal{E}_{x}=\left\{z \in \operatorname{bd}\left(\Omega_{\mathrm{AG}}\right): x \in \mathrm{c} \ell(G \cdot z)\right\}$ is an $N_{x}$-bundle over the closed orbit $G \cdot x$. Thus it is necessary to estimate $\operatorname{dim}_{\mathbb{R}} N_{x}$.

Recall that any two maximal toral Abelian subalgebras of $\mathfrak{q}^{\mathbb{C}}$ are conjugate and therefore the dimension $m$ of one such is an invariant. Since $\mathfrak{a}^{\mathbb{C}}$ is such an algebra, the following is quite useful (see [28]).

Lemma 3.2.5. - The complex codimension in $\mathfrak{q}^{\mathbb{C}}$ of every component of the nilpotent cone in $\mathfrak{q}^{\mathbb{C}}$ is $m$.

Proof of Proposition 3.2.4. - We prove the estimate $\operatorname{codim}_{\Omega} \mathcal{E}_{x} \geq \operatorname{dim} \mathfrak{a}$. For this observe that, since $G \cdot s$ is closed in $\operatorname{Aut}_{\mathbb{R}}\left(\mathfrak{g}^{\mathbb{C}}\right)$, an application of the Luna slice theorem for the (closed) complex orbit $G^{\mathbb{C}}$.s in the complexification of $\operatorname{Im}(\eta)$ yields the bundle structure $\operatorname{Im}(\eta)=G \times_{G_{s}} \mathfrak{q}$ locally near $s$; in particular $\operatorname{codim}_{\mathfrak{q}} N_{x}=\operatorname{codim}_{\Omega}\left(\mathcal{E}_{x}\right)$. The result follows from the above Lemma by noting that $\operatorname{codim}_{\mathfrak{q}} N_{x}$ is at most the complex codimension of the nilpotent cone in $\mathfrak{q}^{\mathbb{C}}$ and, as mentioned above, that $\operatorname{dim} \mathfrak{a}=m$.

BULLETIN DE LA SOCIÉTÉ MATHÉMATIQUE DE FRANCE 
The group $S^{\mathbb{C}}$ constructed above for a generic boundary point has the property that the intersection of the $S^{\mathbb{C}}$-orbit, i.e., a 2-dimensional affine quadric $Q_{2} \cong \mathrm{SL}_{2}(\mathbb{C}) / \mathbb{C}^{*}\left(\right.$ or $\left.\cong \mathrm{SL}_{2}(\mathbb{C}) / N\left(\mathbb{C}^{*}\right)\right)$ with $\Omega_{\mathrm{AG}}$ contains an AkhiezerGindikin domain $\Omega_{\mathrm{AG}}^{S L} \cong D \times \bar{D}$ of $Q_{2}$. To see this, we will conjugate $S^{\mathbb{C}}$ by an element of $G$ in order to relate $S^{\mathbb{C}}$ to the fixed Abelian Lie algebra $\mathfrak{a}$. This is carried out in the next section.

3.3. The intersection property. - To complete our task we conjugate the group $S^{\mathbb{C}}$ obtained in $\S 3.1$ above by an element $h$ in the isotropy group $G_{x_{1}}$ so that it can be easily seen that the resulting orbit $Q_{2}=S^{\mathbb{C}} \cdot x_{1}$ intersects $\Omega_{\mathrm{AG}}$ in the Akhiezer-Gindikin domain of $Q_{2}$.

The following is a first step in this direction.

Proposition 3.3.1. - Let $G \cdot \exp E \cdot \exp i A \cdot x_{0}=G \cdot x_{1}$ be any non-closed orbit in $\operatorname{bd}\left(\Omega_{\mathrm{AG}}\right)$ and $(E, H, F)$ a $\mathfrak{s l}_{2}$-triple in $\left(\mathfrak{g}^{\mathbb{C}}\right)^{\eta(\exp i A)}$ as in Lemma 2.2.2. Given $Z:=E-F$, then there exits $h \in G_{x_{1}}$ so that $\operatorname{Ad}(h)(Z) \in i \mathfrak{a}$.

This result is an immediate consequence of the following basic fact.

Lemma 3.3.2. - Let $\mathfrak{l}$ be a real reductive Lie algebra, $\theta$ a Cartan involution and $\sigma$ a further involution which commutes with $\theta$. Let $\mathfrak{l}=\mathfrak{k} \oplus \mathfrak{p}$ be the eigenspace decomposition with respect to $\theta$ and $\mathfrak{l}=\mathfrak{h} \oplus \mathfrak{q}$ with respect to $\sigma$. Then, if $\mathfrak{a} \subset \mathfrak{p} \cap \mathfrak{q}$ is a maximal Abelian subalgebra of $\mathfrak{q}$ and $\xi$ is a hyperbolic semi-simple element of $\mathfrak{q}$, there exists $h \in \operatorname{Int}(\mathfrak{h})$ such that $\operatorname{Ad}(h)(\xi) \in \mathfrak{q}$.

Proof. - Since $\xi$ is hyperbolic, we may assume that there is a Cartan involution $\theta^{\prime}: \mathfrak{l} \rightarrow \mathfrak{l}$ such that $\theta^{\prime}(\xi)=-\xi$ and $\theta^{\prime} \sigma=\sigma \theta^{\prime}$. Then there exists $h \in \operatorname{Int}(\mathfrak{h})$ with $\operatorname{Ad}(h) \theta^{\prime} \operatorname{Ad}\left(h^{-1}\right)=\theta$ (see [31]) and $\operatorname{Ad}(h)(\xi) \in \mathfrak{p} \cap \mathfrak{q}$.

To complete the proof, just note that $(\mathfrak{h} \cap \mathfrak{k}) \oplus(\mathfrak{p} \oplus \mathfrak{q})$ is a Riemannian symmetric Lie algebra where any two maximal Abelian algebras in $\mathfrak{p} \cap \mathfrak{q}$ are conjugate by an element of $\operatorname{Int}(\mathfrak{h} \cap \mathfrak{k})$.

Proof of Proposition 3.3.1. - Observe that $\operatorname{ad}(Z)$ has only imaginary eigenvalues. Replacing $\left(\mathfrak{g}^{\mathbb{C}}\right)^{\eta(\exp i A)}=\mathfrak{h} \oplus \mathfrak{q}$ by the dual $\tilde{\mathfrak{l}}:=\mathfrak{h} \oplus i \mathfrak{q}=\tilde{\mathfrak{h}} \oplus \tilde{\mathfrak{q}}$ and defining $\tilde{\sigma}$ and $\tilde{\theta}$ accordingly, we apply the above Lemma to $\xi:=i Z$ and the Abelian Lie algebra $\mathfrak{a} \subset \tilde{\mathfrak{q}}$ to obtain $h \in \operatorname{Int}(\mathfrak{h})$ with $\operatorname{Ad}(h)(\xi) \in \mathfrak{a}$. Thus $\operatorname{Ad}(h)(Z)$ has the required property $\operatorname{Ad}(h)(Z) \in i \mathfrak{a}$.

We now show that for $z \in \operatorname{bd}_{\text {gen }}\left(\Omega_{\mathrm{AG}}\right)$ the group $S^{\mathbb{C}}$ which is associated to the $\mathfrak{s l}_{2}$-triple constructed in the above proposition produces a $Q_{2}$-slice. For a precise formulation it is convenient to let $\operatorname{bd}_{\text {gen }}\left(\omega_{\mathrm{AG}}\right):=\operatorname{bd}\left(\omega_{\mathrm{AG}}\right) \backslash E$, where $E$ is the union of the lower-dimensional strata as in $\S 3.2$.

Proposition 3.3.3. - For $z \in \operatorname{bd}_{\text {gen }}\left(\Omega_{\mathrm{AG}}\right)$ and $x_{1}=\exp i A \cdot x_{0}$ the associated point with $i A \in \operatorname{bd}_{\text {gen }}\left(i \omega_{\mathrm{AG}}\right)$ it follows that the line $\mathbb{R}(E-F)$ is transversal to $\operatorname{bd}_{\text {gen }}\left(i \omega_{\mathrm{AG}}\right)$ at $i A$ in $i \mathfrak{a}$. 
The proof requires a more explicit description of $\left(\mathfrak{g}^{\mathbb{C}}\right)^{\eta(\exp i A)}=: \mathfrak{l}=\mathfrak{h} \oplus \mathfrak{q}$. For this, recall the root decompositions of $\mathfrak{g}$ and $\mathfrak{g}^{\mathbb{C}}$ with respect to $\mathfrak{a}$ or $\mathfrak{a}^{\mathbb{C}}$, respectively: $\mathfrak{g}^{\mathbb{C}}=C_{\mathfrak{k}}\left(\mathfrak{a}^{\mathbb{C}}\right) \oplus \mathfrak{a}^{\mathbb{C}} \oplus \bigoplus_{\Phi(\mathfrak{a})} \mathfrak{g}_{\lambda}^{\mathbb{C}}$. The behavior of this decomposition with respect to our involutions is the following: $\theta\left(\mathfrak{g}_{\lambda}^{\mathbb{C}}\right)=\mathfrak{g}_{-\lambda}^{\mathbb{C}}$ and $\tau\left(\mathfrak{g}_{\lambda}^{\mathbb{C}}\right)=\mathfrak{g}_{-\lambda}^{\mathbb{C}}$; furthermore, the root decomposition is $\sigma$-stable, i.e., $\sigma\left(\mathfrak{g}_{\lambda}^{\mathbb{C}}\right)=\mathfrak{g}_{\lambda}^{\mathbb{C}}$. Fix a $\tau$-stable basis of root covectors, i.e., select any basis $L_{\lambda}^{1}, \ldots, L_{\lambda}^{k}$ of $\mathfrak{g}_{\lambda}=\left(\mathfrak{g}_{\lambda}^{\mathbb{C}}\right)^{\sigma}$ and define $L_{-\lambda}^{j}:=\tau\left(L_{\lambda}^{j}\right)$. Define $\mathfrak{g}[\lambda]:=\mathfrak{g}_{\lambda} \oplus \mathfrak{g}_{-\lambda}, \mathfrak{g}^{\mathbb{C}}[\lambda]:=\mathfrak{g}[\lambda] \oplus i \mathfrak{g}[\lambda]$ and notice that $\mathfrak{g}[\lambda]=(\mathfrak{g}[\lambda])^{\tau} \oplus(\mathfrak{g}[\lambda])^{-\tau}$. Finally, set

$$
X_{[\lambda]}^{j}:=L_{\lambda}^{j}+L_{-\lambda}^{j}, \quad Y_{[\lambda]}^{j}:=L_{\lambda}^{j}-L_{-\lambda}^{j}
$$

and observe that $X_{[\lambda]}^{j} \in(\mathfrak{g}[\lambda])^{\tau}, Y_{[\lambda]}^{j} \in(\mathfrak{g}[\lambda])^{-\tau}$. The reason for introducing such a basis is that the complex subspaces $\left(\left(X_{[\lambda]}^{j}, Y_{[\lambda]}^{j}\right)\right)_{\mathbb{C}}$ are $\operatorname{Ad}(t)$-stable for any $t:=\exp i A, A \in \mathfrak{a}$.

Express $\operatorname{Ad}(t)$ as a matrix with respect to the basis $X_{[\lambda]}^{j}, Y_{[\lambda]}^{j}$ :

$$
\operatorname{Ad}(t)_{\mid\left(\left(X_{[\lambda]}, Y_{[\lambda]}\right)\right)}=\left(\begin{array}{cc}
\cosh \lambda(i A) & \sinh \lambda(i A) \\
\sinh \lambda(i A) & \cosh \lambda(i A)
\end{array}\right)
$$

Let $\mathfrak{g}^{\mathbb{C}}=\mathfrak{k}^{\mathbb{C}} \oplus \mathfrak{p}^{\mathbb{C}}$ be the complexification of the Cartan decomposition of $\mathfrak{g}$. A simple calculation yields for $t=\exp i A$ :

$$
\begin{gathered}
\mathfrak{h}=\mathfrak{g} \cap \operatorname{Ad}(t)\left(\mathfrak{k}^{\mathbb{C}}\right)=C_{\mathfrak{k}}(\mathfrak{a}) \oplus \bigoplus_{\lambda(A)=\mathbb{Z} \pi} \mathfrak{g}[\lambda]^{\tau} \oplus \bigoplus_{\lambda(A)=\frac{1}{2} \pi+\mathbb{Z} \pi} \mathfrak{g}[\lambda]^{-\tau}, \\
\mathfrak{q}=i \mathfrak{g} \cap \operatorname{Ad}(t)\left(\mathfrak{p}^{\mathbb{C}}\right)=i \mathfrak{a} \oplus \bigoplus_{\lambda(A)=\mathbb{Z} \pi} i \mathfrak{g}[\lambda]^{-\tau} \oplus \bigoplus_{\lambda(A)=\frac{1}{2} \pi+\mathbb{Z} \pi} i \mathfrak{g}[\lambda]^{\tau} .
\end{gathered}
$$

Let $A \in \operatorname{bd}_{\text {gen }}\left(\omega_{\mathrm{AG}}\right)$ be boundary-generic, i.e., there is a single $\lambda \in \Phi(\mathfrak{a})$ with

$$
\lambda(A)= \pm \frac{\pi}{2}, \quad \mu(A) \notin \frac{1}{2} \pi \mathbb{Z} \text { for all } \mu \in \Phi(\mathfrak{a}) \backslash\{ \pm \lambda\} .
$$

The above general formulas imply that the centralizer subalgebra $\left(\mathfrak{g}^{\mathbb{C}}\right)^{\eta(\exp i A)}$ for such a boundary-generic point as above is given by

$$
\left(\mathfrak{g}^{\mathbb{C}}\right)^{\eta(\exp i A)}=\mathfrak{m} \oplus \mathfrak{g}[\lambda]^{-\tau} \oplus i \mathfrak{a} \oplus i \mathfrak{g}[\lambda]^{\tau} .
$$

To complete the proof of the proposition it is then enough to show that for the selected $\mathfrak{s l}_{2}$-triple $(E, H, F) \in\left(\mathfrak{g}^{\mathbb{C}}\right)^{\eta(\exp i A)}$ it follows that $E-F \in \mathbb{R} i h_{\lambda}$, where $h_{\lambda} \in \mathfrak{a}$ is the coroot determined by the root $\lambda \in \Phi(\mathfrak{a})$. This is the content of the following

LEMmA 3.3.4. - Let $A \in\left\{\lambda=\frac{1}{2} \pi\right\} \cap \operatorname{bd}_{\text {gen }}\left(\omega_{\mathrm{AG}}\right)$ be boundary-generic as above. Then $E-F \in \mathbb{R} i h_{\lambda}$. 
Proof. - Let $\mathfrak{l}:=\left(\mathfrak{g}^{\mathbb{C}}\right)^{\eta(\exp i A)}=\mathfrak{h} \oplus \mathfrak{q}$. Since $((E, H, F))_{\mathbb{R}}$ is semi-simple, it follows that $((E, H, F))_{\mathbb{R}} \subset[\mathfrak{l}: \mathfrak{l}]$. Hence, since $B\left(\left[\mathfrak{g}_{\lambda}: \mathfrak{g}_{-\lambda}\right],\{\lambda=0\}\right)=0$ ( $B$ denotes the Killing form) we have

$$
\begin{aligned}
{[\mathfrak{l}: \mathfrak{l}] } & =\left[\mathfrak{m} \oplus \mathfrak{g}[\lambda]^{-\tau} \oplus i \mathfrak{a} \oplus i \mathfrak{g}[\lambda]^{\tau}: \mathfrak{m} \oplus \mathfrak{g}[\lambda]^{-\tau} \oplus i \mathfrak{a} \oplus i \mathfrak{g}[\lambda]^{\tau}\right] \\
& =\mathfrak{m} \oplus \mathbb{R} i h_{\lambda} \oplus \mathfrak{g}[\lambda]^{-\tau} \oplus i \mathfrak{g}[\lambda]^{\tau} .
\end{aligned}
$$

By Proposition 3.3.1 we have $E-F \in i \mathfrak{a}$. Finally, $E-F \in i \mathfrak{a} \cap[\mathfrak{l}: \mathfrak{l}]=\mathbb{R} i h_{\lambda}$.

Recall that the set $\operatorname{bd}_{\text {gen }}\left(\Omega_{\mathrm{AG}}\right)=\operatorname{bd}\left(\Omega_{\mathrm{AG}}\right) \backslash(\mathcal{C} \cup \mathcal{E})$ consists of certain non-closed orbits in the boundary of $\Omega_{\mathrm{AG}}$.

TheOREM 3.3.5. - On every $G$-orbit in $\mathrm{bd}_{\text {gen }}\left(\Omega_{\mathrm{AG}}\right)$ there exists a point of the form $z:=\exp E \cdot \exp i A \cdot x_{0}, A \in \operatorname{bd}_{\text {gen }}\left(\omega_{\mathrm{AG}}\right), E$ nilpotent, and a corresponding 3-dimensional simple subgroup $S^{\mathbb{C}} \subset G^{\mathbb{C}}$ such that

1) the 2-dimensional affine quadric $S^{\mathbb{C}} \cdot \exp i A \cdot x_{0}=: S^{\mathbb{C}} \cdot x_{1}$ contains $z$;

2) the intersection $\Omega_{\mathrm{AG}} \cap S^{\mathbb{C}} \cdot x_{1}$ contains an Akhiezer-Gindikin domain $\Omega_{\mathrm{AG}}(S)$ of $S^{\mathbb{C}} \cdot x_{1}$, i.e., the orbit $S^{\mathbb{C}} \cdot x_{1}$ is a $Q_{2}$-slice.

Proof. - Given a non-closed $G$-orbit in $\operatorname{bd}_{\text {gen }}\left(\Omega_{\mathrm{AG}}\right)$ let $z=\exp E \cdot \exp i A \cdot x_{0}$ be an optimal base point as in Lemma 3.1.1. By Proposition 3.3.1 we may choose an $\mathfrak{s l}_{2}$-triple $(E, H, F)$ in $\left(\mathfrak{g}^{\mathbb{C}}\right)^{\eta(\exp i A)}$ such that $E-F \in i \mathfrak{a}$. Let $S^{\mathbb{C}} \subset G^{\mathbb{C}}$ be the complex subgroup with Lie algebra $\mathfrak{s}^{\mathbb{C}}:=((E, H, F))_{\mathbb{C}}$. By construction $S^{\mathbb{C}} \cdot x_{1}$ contains $z$.

For a boundary-generic point $x_{1}$ with $\lambda(A)=\frac{1}{2} \pi$ and $\mu(A) \neq \frac{1}{2} \pi \mathbb{Z}$ for all $\mu \neq \pm \lambda$ we already know by 3.3.4 that $E-F \in \mathbb{R} i h_{\lambda}$. Assume that $h_{\lambda} \in \mathfrak{a}$ is the normalized coroot of $\lambda$, i.e., $\lambda\left(h_{\lambda}\right)=2$. Since $\omega_{\mathrm{AG}}$ is invariant under the Weyl group, the image $A^{\prime}$ of $A$ under the reflection on $\{\lambda=0\}$ is also boundary-generic, and the intersection of $A-\mathbb{R} h_{\lambda}$ with $\omega_{\mathrm{AG}}$ is the segment $\left\{A-t h_{\lambda}: t \in\left(0, \frac{1}{2} \pi\right)\right\}$ with boundary points $A$ and $A^{\prime}$.

Recall that $(e, H, f)$ with $E=i e$ and $F=-i f$ is an $\mathfrak{s l}_{2}$-triple in $\mathfrak{s}^{\mathbb{C}}$ such that $\mathfrak{s}:=\mathfrak{g} \cap \mathfrak{s}^{\mathbb{C}}=((e, H, f))_{\mathbb{R}}$. Let $S$ denote the corresponding subgroup in $S^{\mathbb{C}}$ (isomorphic to $\mathrm{SL}_{2}(\mathbb{R})$ or $\mathrm{PSL}_{2}(\mathbb{R})$ ). The $S$-isotropy at all points $\exp \left(\left(-\frac{1}{2} \pi, 0\right) i h_{\lambda}+i A\right) \cdot x_{0}$ is compact and it is non-compact at $\exp i A \cdot x_{0}$ and $\exp i A^{\prime} \cdot x_{0}$. Hence, $S \cdot \exp \left(\left(-\frac{1}{2} \pi, 0\right) i h_{\lambda}+i A\right) \cdot x_{0}$ is an Akhiezer-Gindikin domain in $S^{\mathbb{C}} \cdot x_{1}$ which is contained in $\Omega_{\mathrm{AG}}$.

3.4. Domains of holomorphy. - Let $S^{\mathbb{C}}=\mathrm{SL}_{2}(\mathbb{C}), S=\mathrm{SL}_{2}(\mathbb{R})$ be embedded in $S^{\mathbb{C}}$ as the subgroup of matrices which have real entries and let $K_{S}=\mathrm{SO}_{2}(\mathbb{R})$. To fix the notation, let $D_{0}$ and $D_{\infty}$ be the open $S$-orbits in $\mathbb{P}_{1}(\mathbb{C})$. Further, choose $\mathbb{C} \subset \mathbb{C P}^{1}=\mathbb{C} \cup\{\infty\}$ in such a way that $0 \in D_{0}$ and $\infty \in D_{\infty}$ are the $K_{S}$-fixed points.

Now let $S^{\mathbb{C}}$ act diagonally on $Z=\mathbb{C P}^{1} \times \mathbb{C P}^{1}$ and note that the open orbit $\Omega$, which is the complement of the diagonal $\operatorname{diag}\left(\mathbb{C P}^{1}\right)$ in $Z$, is the complex symmetric space $S^{\mathbb{C}} / K_{S}^{\mathbb{C}}$. Note that in $\mathbb{C P}^{1} \times \mathbb{C P}^{1}$ there are four open 
$\mathrm{SL}_{2}(\mathbb{R}) \times \mathrm{SL}_{2}(\mathbb{R})$-orbits: the bi-disks $D_{\alpha} \times D_{\beta}$ for any pair $(\alpha, \beta)$ from $\{0, \infty\}$. As $S$-spaces, the domains $D_{0} \times D_{\infty}$ and $D_{\infty} \times D_{0}$ are equivariantly biholomorphic; further, they are actually subsets of $\Omega$,and the Riemannian symmetric space $S / K_{S}$ sits in each of them as the totally real $S$-orbit $S \cdot(0, \infty)$ (or $S \cdot(\infty, 0)$, respectively). Depending on which of these points is chosen as a reference point in $\Omega$, both domains can be considered as the Akhiezer-Gindikin domain

$$
\Omega_{\mathrm{AG}}=D_{0} \times D_{\infty}=S \cdot \exp i \omega_{\mathrm{AG}} \cdot(0, \infty), \quad D_{\infty} \times D_{0}=S \cdot \exp i \omega_{\mathrm{AG}} \cdot(\infty, 0)
$$

with $\omega_{\mathrm{AG}}=\left(-\frac{1}{4} \pi, \frac{1}{4} \pi\right) h_{\alpha}$ and $h_{\alpha} \in \mathfrak{a}$ is the normalized coroot $\left(i . e ., \alpha\left(h_{\alpha}\right)=2\right)$.

Our main point here is to understand $S$-invariant Stein domains in $\Omega$ which properly contain $\Omega_{\mathrm{AG}}$. By symmetry we may assume that such has non-empty intersection with $D_{0} \times D_{0}$. Observe that $\left(D_{0} \times D_{0}\right) \cap \Omega=D_{0} \times D_{0} \backslash \operatorname{diag}\left(D_{0}\right)$. Furthermore, other than $\operatorname{diag}\left(D_{0}\right)$, all $S$-orbits in $D_{0} \times D_{0}$ are closed real hypersurfaces. For $D_{0} \times D_{0} \backslash \operatorname{diag}\left(D_{0}\right)$ let $\Omega(p)$ be the domain bounded by $S \cdot p$ and $\operatorname{diag}\left(D_{0}\right)$. We shall show that a function which is holomorphic in a neighborhood of $S \cdot p$ extends holomorphically to $\Omega(p)$.

For this, define $\Sigma:=\{(-s, s): 0 \leq s<1\} \subset D_{0} \times D_{0}$. It is a geometric slice for the $S$-action. We say that a (1-dimensional) complex curve $C \subset \mathbb{C}^{2} \subset Z$ is a supporting curve for $\operatorname{bd}(\Omega(p))$ at $p$ if $C \cap \mathrm{c} \ell(\Omega(p))=\{p\}$. Here, $\mathrm{c} \ell(\Omega(p))$ denotes the topological closure in $D_{0} \times D_{0}$.

Proposition 3.4.1. - For every $p \in D_{0} \times D_{0} \backslash \operatorname{diag}\left(D_{0}\right)$ there exists a supporting curve for $\mathrm{bd}(\Omega(p))$ at $p$.

Proof. - Recall that we consider $D_{0}$ embedded in $\mathbb{C}$ as the unit disc. It is enough to construct such a curve $C \subset \mathbb{C}^{2}$ at each point $p_{s}=(-s, s) \in \Sigma, s \neq 0$. For this we define $C_{s}:=\{(-s+z, s+z): z \in \mathbb{C}\}$. To prove $C_{s} \cap c \ell\left(\Omega\left(p_{s}\right)\right)=\left\{p_{s}\right\}$ let $d$ be the Poincare metric of the unit disc $D_{0}$, considered as the function $d: D_{0} \times D_{0} \rightarrow \mathbb{R}_{\geq 0}$. Note that it is an $S$-invariant function on $D_{0} \times D_{0}$. In fact the values of $d$ parameterize the $S$-orbits.

We now claim that $d(-s+z, s+z) \geq d(-s, s)=d\left(p_{s}\right)$ for $z \in \mathbb{C}$ and $(-s+z, s+z) \in D_{0} \times D_{0}$, with equality only for $z=0$, i.e., $C_{s}$ touches $\mathrm{c} \ell\left(\Omega\left(p_{s}\right)\right)$ only at $p_{s}$. To prove the above inequality, it is convenient to compare the Poincaré length of the Euclidean segment $\operatorname{seg}(z-s, z+s)$ in $D_{0}$ with the length of $\operatorname{seg}(-s, s)$. Writing the corresponding integral for the length, it is clear, without explicit calculation, that $d(-s+x, s+x)>d(-s, s)$ for $z=x \in \mathbb{R} \backslash 0$. The same argument shows also that $d(-s+x+i y, s+x+i y)>d(-s+x, s+x)$ for all non-zero $y \in \mathbb{R}$ and the proposition is proved.

From the above construction it follows that the boundary hypersurfaces $S(p)$ are strongly pseudoconvex. Since then the smallest Stein domain containing a $S$-invariant neighborhood of $S(p)$ is $\Omega(p) \backslash \operatorname{diag}\left(D_{0}\right)$, the following is immediate. 
Corollary 3.4.2. - For $p \in D_{0} \times D_{0} \backslash \operatorname{diag}\left(D_{0}\right)$ every function $f$ which is holomorphic on some neighborhood of the orbit $S \cdot p$ extends holomorphically to $\Omega(p) \backslash \operatorname{diag}\left(D_{0}\right)$. An analogous statement is valid for $p \in D_{\infty} \times D_{\infty} \backslash \operatorname{diag}\left(D_{\infty}\right)$.

Observe that the set $b_{g e n}\left(D_{0} \times D_{\infty}\right)$ of generic boundary points, which was introduced in section 4.2 , consists of the two $S$-orbits bd $\left(D_{0}\right) \times D_{\infty} \cup$ $D_{0} \times \operatorname{bd}\left(D_{\infty}\right)$. Let $z \in \operatorname{bd}\left(D_{0}\right) \times D_{\infty}\left(\right.$ or $z \in D_{0} \times \operatorname{bd}\left(D_{\infty}\right)$ be such a boundary point.

Corollary 3.4.3. - Let $\widehat{\Omega} \subset Q_{2} \subset \mathbb{C P}^{1} \times \mathbb{C P}^{1}$ be an $S$-invariant Stein domain which contains $D_{0} \times D_{\infty}$ and the boundary point $z$. Then $\widehat{\Omega}$ also contains $D_{0} \times \mathbb{C P}^{1} \backslash \operatorname{diag}\left(\mathbb{C P}^{1}\right)\left(\right.$ or $\mathbb{C P}^{1} \times D_{\infty} \backslash \operatorname{diag}\left(\mathbb{C P}^{1}\right)$, respectively $)$.

Proof. - Let $B$ be a ball around $z$ which is contained in $\widehat{\Omega}$. For $p$ in $B(z) \cap D_{\infty} \times D_{\infty}$ sufficiently close to $z$ it follows that $S \cdot q \subset \widehat{\Omega}$ for all $q$ in $B(z) \cap\left(D_{\infty} \times D_{\infty}\right)$. The result then follows from the previous corollary.

If $\widehat{\Omega}$ is as in the above corollary, the fibers of the projection of $\widehat{\Omega} \subset \mathbb{C P}^{1} \times \mathbb{C P}^{1}$ $\rightarrow \mathbb{P}^{1}$ can be regarded as non-constant holomorphic curves $f: \mathbb{C} \rightarrow \widehat{\Omega}$. One says that a complex manifold $X$ is Brody hyperbolic if there are no such curves.

Corollary 3.4.4. - If $\widehat{\Omega}$ is as above, then $\widehat{\Omega}$ is not Brody hyperbolic.

A complex manifold $X$ is said to be Kobayashi hyperbolic whenever the Kobayashi pseudo-metric is in fact a metric (see [27]). The pseudo-metric is defined in such a way that, if there exists a non-constant holomorphic curve $f: \mathbb{C} \rightarrow X$, then $X$ is not hyperbolic, i.e., Kobayashi hyperbolicity is a stronger condition than Brody hyperbolic. For an arbitrary semi-simple group $G$ the domain $\Omega_{\mathrm{AG}}$ is indeed Kobayashi hyperbolic ( $c f$. [22], see $\S 5$ for stronger results).

The following is our main application of the existence of $Q_{2}$-slices at generic points of $\mathrm{bd}\left(\Omega_{\mathrm{AG}}\right)$.

Theorem 3.4.5. - A G-invariant, Stein and Brody hyperbolic domain $\widehat{\Omega}$ in $G^{\mathbb{C}} / K^{\mathbb{C}}$ which contains $\Omega_{\mathrm{AG}}$ is equal to $\Omega_{\mathrm{AG}}$.

Proof. - Arguing by contraposition, if $\Omega_{\mathrm{AG}}$ is strictly contained in a $G$ invariant Stein domain $\widehat{\Omega}$, then by Theorem 3.3.5 there exists a $Q_{2}$-slice at a generic boundary point $z \in \operatorname{bd}\left(\Omega_{\mathrm{AG}}\right) \cap \widehat{\Omega}$ with $Q_{2} \cap \widehat{\Omega}$ an $S$-invariant Stein domain properly containing the Akhiezer-Gindikin domain of $Q_{2}$. However, by Corollary 3.4.4, such a domain in $Q_{2}$ is not Brody hyperbolic.

\section{Hyperbolicity and the characterization of cycle domains}

In this section it is shown the Wolf cycle domains $\Omega_{W}(D)$ are Kobayashi hyperbolic. The above theorem then yields their characterization (see 4.2.5). 
4.1. Families of hyperplanes. - We start by proving a general result concerning families of hyperplanes in projective space and their intersections with locally closed subvarieties. Since such a subvariety is usually regarded as being embedded by sections of some line bundle, it is natural to regard the projective space as the projectivization $\mathbb{P}\left(V^{*}\right)$ of the dual space and a hyperplane in $\mathbb{P}\left(V^{*}\right)$ as a point in $\mathbb{P}(V)$.

We will think of a subset $S \in \mathbb{P}(V)$ as parameterizing a family of hyperplanes in $\mathbb{P}\left(V^{*}\right)$. A non-empty subset $S \subset \mathbb{P}(V)$ is said to have the normal crossing property if for every $k \in \mathbb{N}$ there exist $H_{1}, \ldots H_{k} \in S$ so that for every subset $I \subset\{1, \ldots, k\}$ the intersection $\bigcap_{i \in I} H_{i}$ is $|I|$-codimensional. If $|I| \geq \operatorname{dim}_{\mathbb{C}} V$, this means that the intersection is empty.

In the sequel $\langle S\rangle$ denotes the complex linear span of $S$ in $\mathbb{P}(V)$, i.e., the smallest plane in $\mathbb{P}(V)$ containing $S$.

Proposition 4.1.1. - A locally closed, irreducible real analytic subset $S$ with $\langle S\rangle=\mathbb{P}(V)$ has the normal crossing property.

Proof. - We proceed by induction over $k$. For $k=1$ there is nothing to prove. Given a set $\left\{H_{s_{1}}, \ldots, H_{s_{k}}\right\}$ of hyperplanes with the normal crossing property and a subset $I \subset\left\{s_{1}, \ldots, s_{k}\right\}$, define

$$
\Delta_{I}:=\bigcap_{s \in I} H_{s}, \quad \mathcal{H}(I):=\left\{s \in S: H_{s} \supset \Delta_{I}\right\}, \quad \mathcal{C} \ell_{k}:=\bigcup_{\substack{J \subset\left\{s_{1}, \ldots, s_{k}\right\} \\ \Delta J \neq \varnothing}} \mathcal{H}(J) .
$$

We wish to prove that $S \backslash \mathcal{C} \ell_{k} \neq \varnothing$. For this, note that each $\mathcal{H}(I)$ is a real analytic subvariety of $S$. Hence, if $S=\mathcal{C} \ell_{k}$, then $S=\mathcal{H}(J)$ for some $J$ with $\Delta_{J} \neq \varnothing$. However, $\left\{H \in \mathbb{P}\left(V^{*}\right): H \supset \Delta_{J}\right\}$ is a proper, linear plane $\mathcal{L}(J)$ of $\mathbb{P}(V)$. Consequently, $S \subset \mathcal{L}(J)$, and this would contradict $\langle S\rangle=\mathbb{P}(V)$. Therefore, there exists $s \in S \backslash \mathcal{C} \ell_{k}$, or equivalently, $\left\{H_{s_{1}}, \ldots, H_{s_{k}}, H_{s}\right\}$ has the normal crossing property.

It is known that if $H_{1}, \ldots, H_{2 m+1}$ are hyperplanes having the normal crossing property, where $m=\operatorname{dim}_{\mathbb{C}} \mathbb{P}(V)$, then $\mathbb{P}\left(V^{*}\right) \backslash \bigcup H_{j}$ is Kobayashi hyperbolic (cf. [12], see also [27, p. 137]).

Corollary 4.1.2. - If $S$ is a locally closed, irreducible and generating real analytic subset of $\mathbb{P}(V)$, then there exist hyperplanes $H_{1}, \ldots H_{2 m+1} \in S$ so that the complement $\mathbb{P}\left(V^{*}\right) \backslash \bigcup H_{j}$ is Kobayashi hyperbolic.

Our main application of this result arises in the case where $S$ is an orbit of the real form at hand.

Corollary 4.1.3. - Let $G^{\mathbb{C}}$ be a reductive complex Lie group, $G$ a real form, $V^{*}$ an irreducible $G^{\mathbb{C}}$-representation space and $S$ a $G$-orbit in $\mathbb{P}(V)$. Then there exist hyperplanes $H_{1}, \ldots, H_{2 m+1} \in S$ so that $\mathbb{P}\left(V^{*}\right) \backslash \bigcup H_{j}$ is Kobayashi hyperbolic. 
Proof. - From the irreducibility of the representation $V^{*}$, it follows that $V$ is likewise irreducible and this, along with the identity principle, implies that for $\langle S\rangle=\mathbb{P}(V)$.

4.2. Hyperbolic domains in $\boldsymbol{G}^{\mathbb{C}} / \boldsymbol{K}^{\mathbb{C}}$. - Hypersurfaces $H$ in $\Omega=G^{\mathbb{C}} / K^{\mathbb{C}}$ which are invariant under the action of an Iwasawa-Borel group $B$, i.e., Borel groups which contain the $A N$ part of some Iwasawa-decomposition $G=K A N$, play a key role in the study of $G$-invariant domains (see also [22], [23], [24] and [25]). In the sequel we shall simply refer to such $H$ simply as a $B$-hypersurface.

Recall that if $H_{1}, \ldots, H_{m}$ are all of the irreducible $B$-hypersurfaces in $G^{\mathbb{C}} / K^{\mathbb{C}}$ and if $\bigcup_{j}\left(\bigcup_{g \in G} g\left(H_{j}\right)\right)$ is removed from $\Omega$, then the connected component $\Omega_{I}$ of the resulting domain is the Akhiezer-Gindikin domain $\Omega_{\mathrm{AG}}$ (see [22], [2] and [33]). In particular, the $\Omega_{I}$ is non-empty.

Now if $H$ is just one (possibly not irreducible) $B$-hypersurface, then the set $\bigcup_{g \in G} g(H)=\bigcup_{g \in K} g(H)$ is closed and its complement in $\Omega=G^{\mathbb{C}} / K^{\mathbb{C}}$ is open. Let $\Omega_{H}$ be the connected component of that open complement, containing the chosen base point $x_{0}$. It is likewise a non-empty $G$-invariant Stein domain in $\Omega=G^{\mathbb{C}} / K^{\mathbb{C}}$.

Here we shall prove that, if $G$ is not Hermitian, any such $\Omega_{H}$ is Kobayashi hyperbolic. In the Hermitian case one easily describes the situation where $\Omega_{H}$ is not hyperbolic.

Let $H$ be given as above and let $L$ be the line bundle which it defines. Let $\sigma_{H}$ be the corresponding section, i.e., $\left\{\sigma_{H}=0\right\}=H$.

Note that $\sigma_{H}$ is a $B$-eigenvector in $\Gamma(\Omega, L)$. Let $V_{H} \subset \Gamma(\Omega, L)$ be the irreducible $G^{\mathbb{C}}$-representation space which contains $\sigma_{H}$. Define $\varphi_{H}: \Omega \rightarrow$ $\mathbb{P}\left(V_{H}^{*}\right)$ to be the canonically associated $G^{\mathbb{C}}$-equivariant meromorphic map.

Lemma 4.2.1. - The map $\varphi_{H}: \Omega \rightarrow \mathbb{P}\left(V_{H}^{*}\right)$ is a regular morphism onto a quasi-projective $G^{\mathbb{C}}$-orbit $G^{\mathbb{C}} \cdot v_{0}^{*}=: \widetilde{\Omega}$.

Proof. - By definition $\varphi_{H}$ is $G^{\mathbb{C}}$-equivariant; in particular its set $E$ of base points is $G^{\mathbb{C}}$-invariant. Since $\Omega$ is $G^{\mathbb{C}}$-homogeneous, $E=\varnothing$.

By definition every section $s \in V_{H}$ is the pull-back $\varphi_{H}^{*}(\widetilde{s})$ of a hyperplane section. Thus, there is a uniquely defined $B$-hypersurface $\widetilde{H}$ in $\mathbb{P}\left(V_{H}^{*}\right)$ with $\varphi_{H}^{-1}(\widetilde{H})=H$. Let $\widetilde{\Omega}_{\widetilde{H}} \subset \mathbb{P}\left(V_{H}^{*}\right)$ be defined analogously to $\Omega_{H}$, i.e., $\widetilde{\Omega}_{\widetilde{H}}=\mathbb{P}\left(V_{H}^{*}\right) \backslash \bigcup_{g \in G} g(\widetilde{H})$. Applying Corollary 4.1.3 to $\mathbb{P}\left(V_{H}^{*}\right)$ and $S:=G \cdot \widetilde{H} \subset \mathbb{P}\left(V_{H}\right)$, it follows that the domain $\widetilde{\Omega}_{\widetilde{H}}$ is Kobayashi hyperbolic. Further, the connected component of $\varphi_{H}^{-1}\left(\widetilde{\Omega}_{\widetilde{H}}\right)$ which contains the base point $x_{0}$ is just the original domain $\Omega_{H}$.

If $\varphi$ has positive dimensional fibers, which indeed can happen in the Hermitian case, then, since the connected components of its fibers contain many holomorphic curves $f: \mathbb{C} \rightarrow \Omega$, it follows that $\Omega_{H}$ is not Kobayashi hyperbolic. 
In the case of finite fibers, since preimages under locally biholomorphic maps of hyperbolic manifolds are hyperbolic, the opposite is true.

TheOrem 4.2.2. - If the $\varphi_{H}$-fibers are finite, then $\Omega_{H}$ is Kobayashi hyperbolic.

Corollary 4.2.3. - If $G$ is not of Hermitian type, then $\Omega_{H}$ is Kobayashi hyperbolic.

Proof. - If $G$ is not of Hermitian type, then $K^{\mathbb{C}}$ is dimension theoretically maximal in $G^{\mathbb{C}}$ and, since $\varphi_{H}$ is non-constant, it follows that it has finite fibers.

Theorem 4.2.4. - The Wolf cycle domain $\Omega_{W}(D)$ of an open orbit $D$ of an arbitrary real form $G$ of an arbitrary complex semi-simple group $G^{\mathbb{C}}$ in an arbitrary flag manifold $Z=G^{\mathbb{C}} / Q$ ist Stein and Kobayashi hyperbolic.

Proof. - It was shown in [25] that every Wolf cycle space $\Omega_{W}(D)$ is the intersection of certain of the $\Omega_{H}$. In the notation of [25] such an intersection is referred to as the associated Schubert domain $\Omega_{S}(D)$. Thus the cycle domains $\Omega_{W}(D)$ are Stein.

If $G$ is not of Hermitian type, then, since it is contained in $\Omega_{H}$ for certain $B$-hypersurfaces $H$, Corollary 4.2 .3 implies that it is hyperbolic.

If $G$ is of Hermitian type, then $\Omega_{W}(D)$ is either the associated bounded symmetric domain $\mathcal{B}$, its complex conjugate or, if $\Omega$ is non-compact, $\mathcal{B} \times \overline{\mathcal{B}}$ (see [43], [45], [46], [25]). Since bounded domains are hyperbolic, this completes the proof.

We now give a characterization of all Wolf cycle domains, including the few exceptions mentioned above. For this recall that $D$ is an open $G$-orbit in $Z=G^{\mathbb{C}} / Q, C_{0}$ the base cycle in $D$, and $G^{\mathbb{C}} \cdot C_{0}=\Omega$ is the corresponding orbit in the cycle space $\mathcal{C}^{q}(Z)$.

TheOREM 4.2.5. - If $\Omega$ is compact, then either $\Omega_{W}(D)$ consists of a single point or $G$ is Hermitian and $\Omega_{W}(D)$ is either the associated bounded symmetric domain $\mathcal{B}$ or its complex conjugate $\overline{\mathcal{B}}$. If $\Omega$ is non-compact, then, regarding $\Omega_{W}(D)$ as a domain $G^{\mathbb{C}} / K^{\mathbb{C}}$, it follows that

$$
\Omega_{W}(D)=\Omega_{\mathrm{AG}}
$$

for every open $G$-orbit in every $G^{\mathbb{C}}$-flag manifold $Z=G^{\mathbb{C}} / Q$.

Proof. - The exceptional case where $\Omega$ is compact is discussed in detail in the proof of Theorem 4.2.4 and therefore we restrict here to the non-compact case.

The statement $\Omega_{W}(D)=\Omega_{S}(D)$ is proved in [25]. In [22] and [33] it is proved that $\Omega_{A G} \subset \Omega_{I}$. By definition $\Omega_{S}(D) \supset \Omega_{I}=\Omega_{\mathrm{AG}}$. Since $\Omega_{W}(D)$ is Stein and hyperbolic (Theorem 4.2.4), by Theorem 3.4.5 it follows that $\Omega_{W}(D)=\Omega_{\mathrm{AG}}$, and all equalities are forced.

BULLETIN DE LA SOCIÉTÉ MATHÉMATIQUE DE FRANCE 
REMARK. - The second author's proof [22] of the inclusion $\Omega_{A G} \subset \Omega_{I}$ only used the existence of a $G$-invariant strictly plurisubharmonic function on $\Omega_{A G}$. It is in fact necessary to use the existence, shown in [10], of such a function which in addition restricts to and exhaustion of $\exp \left(i \omega_{A G}\right) \cdot x_{0}$. In the meantime T. Matsuki [33] has given an algebraic proof which holds in greater generality.

\section{BIBLIOGRAPHY}

[1] Akhiezer (D.) \& Gindikin (S.) - On the Stein extensions of real symmetric spaces, Math. Ann., t. 286 (1990), pp. 1-12.

[2] BARchini (L.) - Stein extensions of real symmetric spaces and the geometry of the flag manifold, Math. Ann., t. 326 (2003), pp. 331-346.

[3] Barchini (L.), Gindikin (S.) \& Wong (H.W.) - Geometry of flag manifolds and holomorphic extensions of Szegö kernels for $\mathrm{SU}(p, q)$, Pacific J. Math., t. 179 (1997), pp. 201-220.

[4] Barchini (L.), Leslie (C.) \& Zierau (R.) - Domains of holomorphy and representations of $\mathrm{Sl}(n, \mathbb{R})$, Manuscripta Math., t. 6 (2001), no. 4, pp. 411-427.

[5] Barlet (D.) \& Koziarz (V.) - Fonctions holomorphes sur l'espace des cycles: la méthode d'intersection, Math. Research Letters, t. 7 (2000), pp. 537-550.

[6] Barlet (D.) \& Magnusson (J.) - Intégration de classes de cohomologie méromorphes et diviseurs d'incidence, Ann. Sci. École Norm. Sup., t. 31 (1998), pp. 811-842.

[7] Birkes (D.) - Orbits of linear algebraic groups, Ann. of Math., t. 93 (1971), no. 2, pp. 459-475.

[8] Bourbaki (N.) - Éléments de mathématique: Groupes et algèbres de Lie, Chapitres 7 et 8, Hermann, Paris, 1975.

[9] Bremigan (R.) - Quotients for algebraic group actions over nonalgebraically closed fields, J. reine angew. Math., t. 453 (1994), pp. 21-47.

[10] Burns (D.), Halverscheid (St.) \& Hind (R.) - The geometry of Grauert tubes and complexification of symmetric spaces, Duke J. Math., t. 118 (2003), pp. 465-491.

[11] CRittenden (R.J.) - Minimum and conjugate points in symmetric spaces, Canad. J. Math., t. 14 (1962), pp. 320-328.

[12] Dufresnoy (H.) - Théorie nouvelle des familles complexes normales. Application à l'étude des fonctions algébrö̈des, Ann. Sci. École Norm. Sup., t. 61 (1944), pp. 1-44.

[13] Dunne (E.G.) \& Zierau (R.) - Twistor theory for indefinite Kähler symmetric spaces, Contemp. Math., t. 154 (1993), pp. 117-132.

TOME $133-2005-\mathrm{N}^{\mathrm{O}} 1$ 
[14] FARAut (J.) - Fonctions sphériques sur un espace symétrique ordonné de type Cayley, in Representation theory and harmonic analysis (Cincinnati, OH, 1994), vol. 191, 1995, pp. 41-55.

[15] Fels (G.) - A note on homogeneous locally symmetric spaces, Transform. Groups, t. 2 (1997), pp. 269-277.

[16] Gindikin (G.) - Tube domains in Stein symmetric spaces, in Positivity in Lie theory: Open problems, W. de Gruyter, 1998, pp. 81-98.

[17] Gindikin (S.) \& Matsuki (T.) - Stein extensions of Riemann symmetric spaces and dualities of orbits on flag manifolds, MSRI-Preprint 2001-028.

[18] HeIer (G.) - Die komplexe Geometrie des Periodengebietes der K3Flächen, Diplomarbeit, Ruhr-Universität Bochum, 1999.

[19] Heinzner (P.) - Equivariant holomorphic extensions of real-analytic manifolds, Bull. Soc. Math. France, t. 121 (1993), pp. 101-119.

[20] Heinzner (P.), Huckleberry (A.) \& Kutzschebauch (F.) - A real analytic version of Abels' Theorem and complexifications of proper Lie group actions, in Complex Analysis and Geometry, Lecture Notes in Pure and Applied Mathematics, Marcel Decker, 1995, pp. 229-273.

[21] Helgason (S.) - Differential geometry, Lie groups, and symmetric spaces, Pure and Applied Mathematics, vol. 80, Academic Press, 1978.

[22] HuCKLEBerRy (A.) - On certain domains in cycle spaces of flag manifolds, Math. Ann., t. 323 (2002), pp. 797-810.

[23] Huckleberry (A.) \& Simon (A.) - On cycle spaces of flag domains of $\mathrm{SL}_{n}(\mathbb{R})$, J. reine angew. Math., t. 541 (2001), pp. 171-208.

[24] Huckleberry (A.) \& Wolf (J.A.) - Cycle spaces of real forms of $\mathrm{SL}_{n}(\mathbb{C})$, in Complex Geometry: A Collection of Papers Dedicated to Hans Grauert', Springer-Verlag, 2002, to appear.

[25] _ Schubert varieties and cycle spaces, Duke J. Math., t. 120 (2003), pp. 229-249.

[26] Humphreys (J.E.) - Linear algebraic groups, Graduate Text in Math., vol. 21, Springer, 1975.

[27] Kobayashi (S.) - Hyperbolic complex spaces, vol. 318, Springer, 1998.

[28] Kostant (B.) \& Rallis (S.) - Orbits and representations associated with symmetric spaces, Amer. J. Math., t. 93 (1971), pp. 753-809.

[29] Krötz (B.) \& Stanton (R.J.) - Holomorphic extensions of representations, I and II, automorphic functions, preprints.

[30] Kutzschebauch (F.) - Eigentliche Wirkungen von Liegruppen auf reellanalytischen Mannigfaltigkeiten, Schriftenreihe des Graduiertenkollegs Geometrie und Mathematische Physik, vol.5, Ruhr-Universität Bochum, 1994.

[31] Matsuki (T.) - The orbits of affine symmetric spaces under the action of minimal parabolic subgroups, J. Math. Soc. Japan, t. 312 (1979), pp. 331357.

BULlETIN DE LA SOCIÉTÉ MATHÉMATIQUE DE FRANCE 
[32] _ Double coset decompositions of redutive Lie groups arising from two involutions, J. Alg., t. 197 (1997), pp. 49-91.

[33] - Preprint.

[34] NovaK (J.D.) - Parameterizing maximal compact subvarieties, Proc. Amer. Math. Soc., t. 124 (1996), pp. 969-975.

[35] Ólafsson (G.) - Analytic continuation in representation theory and harmonic analysis, in Global analysis and harmonic analysis (MarseilleLuminy, 1999), Séminaires \& Congrès, vol. 4, Soc. Math. France, 2000, pp. 201-233.

[36] Ólafsson (G.) \& CRsted (B.) - Analytic continuation of FlenstedJensen representations, Manuscripta Math., t. 74 (1992), no. 1, pp. 5-23.

[37] Onishchik (A.) - Topology of Transitive Transformation Groups, Johann Ambrosius Barth, Leipzig Berlin, 1994.

[38] Patton (C.M.) \& Rossi (H.) - Unitary structures on cohomology, Trans. Amer. Math. Soc., t. 290 (1985), pp. 235-258.

[39] Schwarz (G.) - Lifting smooth homotopies of orbit spaces, Inst. Hautes Études Sci. Publ. Math., t. 50 (1980), pp. 37-135.

[40] - The topology of algebraic quotients, in Topological methods in algebraic transformation groups (New Brunswick, NJ, 1988), Progr. Math., vol. 80, Birkhäuser, 1989, pp. 135-151.

[41] Wells (R.O.) - Parameterizing the compact submanifolds of a period matrix domain by a Stein manifold, in Symposium on Several Complex Variables, Lecture Notes in Math., vol. 184, Springer, 1971, pp. 121-150.

[42] Wolf (J.A.) - The action of a real semi-simple group on a complex flag manifold, I: Orbit structure and holomorphic arc components, Bull. Amer. Math. Soc., t. 75 (1969), pp. 1121-1237.

[43] _ The Stein condition for cycle spaces of open orbits on complex flag manifolds, Ann. of Math., t. 136 (1992), pp. 541-555.

[44] _ Real groups transitive on complex flag manifolds, Proc. Amer. Math. Soc., t. 129 (2001), pp. 2483-2487.

[45] Wolf (J.A.) \& Zierau (R.) - Linear cycle spaces in flag domains, Math. Ann., t. 316 (2000), no. 3, pp. 529-545.

[46] _ The linear cycle space for groups of hermitian type, J. Lie Theory, t. 13 (2003), pp. 189-191. 